\title{
Measurement of Noise Produced by a Plasma Contactor Operating in Ground Based Facilities
}

Steve Snyder

Colorado State University

Fort Collins, Colorado

March 1996

Prepared for

Lewis Research Center

Under Grant NAG3-1700

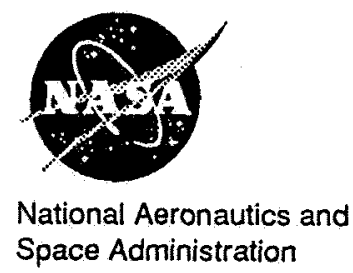





\section{TABLE OF CONTENTS}

Title

Page

I. INTRODUCTION 1

II. EXPERIMENTAL APPARATUS AND PROCEDURES 11

III. EXPERIMENTAL RESULTS AND DISCUSSION 24

Antenna Measurement Methods in a Plasma $\quad 24$

Influence of Vacuum-Chamber

Walls on RE Measurements 33

Noise Induced by Power Supplies $\quad 40$

Relationship Between RE and CE 45

Electromagnetic Wave Broadcast Experiments $\quad 48$

Induced Current Fluctuation Experiments $\quad 54$

Plasma Exclusion Region Experiments 56

Comparison of Bell-Jar and

Vacuum-Tank Results $\quad 60$

IV. CONCLUSIONS AND RECOMMENDATIONS 66

$\begin{array}{lll}\text { V. FUTURE WORK } & 68\end{array}$

$\begin{array}{lll}\text { VI. REFERENCES } & 70\end{array}$

$\begin{array}{ll}\text { APPENDIX A } \begin{array}{c}\text { Measurement of Broadcast Antenna } \\ \text { Radiation Pattern }\end{array} & 73\end{array}$ 


\section{LIST OF FIGURES}

Figure $\quad$ Page

1 Categorization of Electrical Noise. $\quad 4$

2 Sources of Noise in a Plasma Detectable by a Monopole Antenna. 6

3 Categorization of Radiated Emissions. $\quad 7$

4 Vacuum-Tank Facility Schematic -- Normal Configuration. 12

$5 \quad$ Vacuum-Tank Facility Schematic -- Altered Configuration. 13

6 Plasma Contactor and Simulator Schematics. 15

$7 \quad$ Electrical Schematic. $\quad 16$

$8 \quad$ Bell-Jar Facility Schematic. $\quad 18$

9 Typical Clamped-Mode Radiated Emissions. 22

10 Effects of Ambient Plasma on Antenna Response with Monopole Exposed to Plasma. 25

11 Effects of Ambient Plasma on Antenna Response with Monopole Isolated from Plasma.

12 Effects of Ambient Plasma on Antenna Response with Baseplate Isolated from Plasma. 29

13 Effects of Monopole Length on Antenna Measurements. 32

14 Behavior of Electromagnetic Waves in Vacuum-Tank Facility -Broadcast Antenna Aligned on Tank Centerline.

15 Behavior of Electromagnetic Waves in Vacuum-Tank Facility -Broadcast Antenna off of Tank Centerline. 
Figure

16 Effects of Conducting Surface Surrounding Plasma Contactor

in Bell-Jar Test -- Clamped Mode.

17 Effects of Conducting Surface Surrounding Plasma Contactor in Bell-Jar Test -- Idle Mode.

18 Effects of Power Supplies on Clamped-Mode Radiated Emissions.

19 Effects of Power Supplies on Idle-Mode Radiated Emissions.

20 Effects of Power Supplies on Conducted Emissions. 44

21 Typical Conducted and Radiated Emissions in Vacuum-Tank Facility. 46

22 Comparison of Simultaneously-Acquired Raw Data Signals. 47

23 Propagation of Electromagnetic Waves Through Contactor Plasma. 49

24 Attenuation of $100 \mathrm{kHz}$ Signal by Contactor Plasma in Vacuum Tank. 52

25 Coupling of Electromagnetic Waves in Contactor

Plasma to Current Return Line.

26 Coupling of Current Fluctuations in Return Line to Contactor Plasma.

27 Effects of Plasma Exclusion Region Size on

Clamped-Mode Radiated Emissions.

28 Effects of Plasma Exclusion Region Size on Idle-Mode Radiated Emissions.

29 Comparison of Idle-Mode Radiated Emissions

Acquired in Each Facility.

30 Comparison of Clamped-Mode Radiated Emissions

Acquired in Each Facility.

$\begin{array}{lll}\text { A1 Broadcast Antenna Radiation Pattern Measurement Configurations. } & 74\end{array}$

A2 Broadcast Antenna Radiation Patterns. 


\section{INTRODUCTION}

A hollow-cathode based plasma contactor has been selected for use on International Space Station Alpha (ISSA) to control the electrical potential of the spacecraft structure. A plasma contactor is a device which ionizes an expellant gas and creates a localized plasma plume which can establish an electrical connection to another plasma such as the ambient space plasma. Through this connection the contactor may either emit electrons to or collect them from the ambient space plasma, thereby controlling the potential of the spacecraft to which it is connected. The hollow-cathode plasma contactor has many features, described elsewhere $[1,2]$, which make it attractive for spacecraft potential control. Detailed theoretical models of space-station interaction with the Earth's ionosphere predict that the contactor will typically have to emit ampere-level currents to maintain the desired spacecraft potential [3].

A critical design requirement for any electrical device on ISSA, including the plasma contactor, is that it not create electrical noise that can adversely affect the operation of other electrical and electronic equipment. Electrical noise is defined as "unwanted signals that produce undesirable effects in the circuits of the control systems in which they occur" [4]. Noise is random, spontaneous fluctuations resulting from the fundamental physics of electrical devices [5] and hence must be 
analyzed statistically. It limits the performance of electrical systems and it can have fatal consequences in medical and military applications [6]. Operation of the plasma contactor might generate types of noise typically found in electrical circuits and also noise peculiar to plasma sources.

Some kinds of electrical noise commonly found in conventional electrical circuits are thermal noise, shot noise, and flicker noise. Thermal noise is due to the random electron motions in a conductive material, e.g. a resistor, because of its finite temperature. Statistically, at any point in time there may be more electrons at one end of the resistor than the other, hence a voltage difference and a current will exist in the resistor. This thermal noise is measurable and has a well-defined root-meansquare value $[5,7]$. Shot noise is a result of discontinuous current flow, i.e. pulses of current caused by the uneven flow of discrete charges. It is associated with current flow across a potential barrier and hence is observed in diodes and vacuum tubes, for example $[5,7]$. Flicker noise; also known as excess, contact, or $1 / f$ noise; results from the fluctuating conductivity of a device through which a direct current is flowing. First observed in vacuum tubes, it is also seen in composition resistors, diodes, and light sources $[5,7]$. The hollow-cathode plasma contactor could be expected to produce any of these types of noise.

Perhaps the most common examples of noise caused by plasma phenomena are natural, e.g. lightning, sunspots, and cosmic noise. On Earth, plasma noise from gas discharge tubes has historically been a popular and reliable noise source for testing electrical systems [7]. Plasma noise associated with hollow cathode operation may be caused by many different mechanisms in addition to those mentioned above. There 
may be natural fluctuations in the plasma potential, the density of ions and electrons, and also in the mean electromagnetic fields that exist in the plasma. These fluctuations may be independent of, or may be related to, various types of waves which can propagate in a plasma. These waves may be standard electromagnetic waves or plasma waves such as ion acoustic waves or electrostatic waves $[8,9]$. Extensive discussions of plasma waves may be found in refs. 8 and 9 .

Electrical noise may be separated into two groups as suggested in Fig. 1: conducted emissions (CE) and radiated emissions (RE). Radiated emissions may be further classified as either far-field or near-field radiation. Far-field radiation exists at $D \gg \lambda / 2 \pi$, where $D$ is the distance through which the noise is radiated, and $\lambda$ is the wavelength of the radiation. The near-field exists at $\mathrm{D} \approx \lambda / 2 \pi$ and $\mathrm{D} \ll \lambda / 2 \pi$ $[10,11,12,13]$.

All types of noise which may be present in a system can be classified as RE or CE. There are four coupling mechanisms, or path classifications, by which electromagnetic energy, including noise, can be transferred: 1) conductive (via direct contact), 2) capacitive (via electric fields), 3) inductive (via magnetic fields), and 4) radiative (via transverse electromagnetic waves) [10]. Conducted emissions couple directly along a solid conductive medium, such as an electrical wire, as current fluctuations. Radiated emissions, on the other hand, couple through a diffuse environment such as space $[4,13]$. In the case of near-field radiation, coupling occurs either capacitively or inductively. At the frequencies of interest in this study, the radiation will be entirely near-field, i.e. there will be no TEM waves which, by definition, exist only in the far field [12]. 


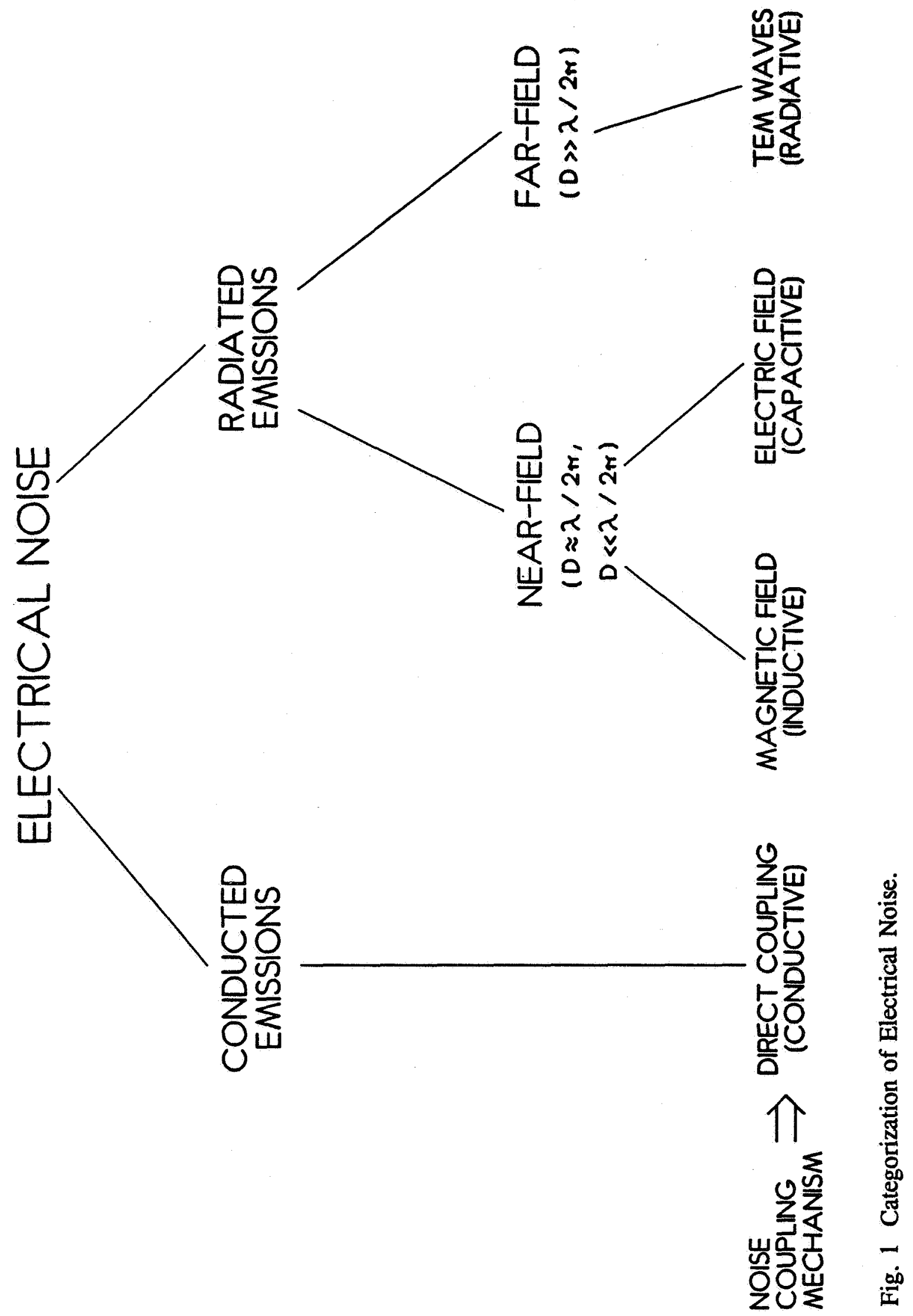


Special difficulties are encountered when attempting to measure RE produced by an operating plasma contactor. The plasma plume created by the contactor expands out into space to a size many times larger than the contactor itself $[3,14]$ and it may thereby envelop potential victim equipment. In ground-based experiments, the plasma plume will spread to fill a vacuum chamber, and any instrument within the chamber that is used to measure RE, such as an antenna, will necessarily be immersed in this plume.

In this study, a monopole antenna was used to sense RE produced by the plasma contactor. The monopole antenna directly measures only electric-field fluctuations, thus the noise sensed in a vacuum chamber by the antenna might come from any of the three sources depicted in Fig. 2. First, the noise may be near-field EM waves which are produced some distance from the antenna either at the contactor (source \#1) or by the plasma (source \#2) and must pass through the plasma to reach the antenna. On the other hand, the noise may be electric-field fluctuations in the plasma caused by the motion of charged particles (current fluctuations) in the immediate vicinity of the antenna (source \#3). These current fluctuations in the plasma are also known as plasma electrostatic waves [8]. Figure 3 summarizes these three noise sources and the relationships between them. As suggested in the figure, radiated emissions due to current fluctuations (electrostatic waves) that occur adjacent to the antenna will be designated locally-produced noise. Radiated emissions due to remote, near-field EM waves will be designated remotely-produced noise in this report. 


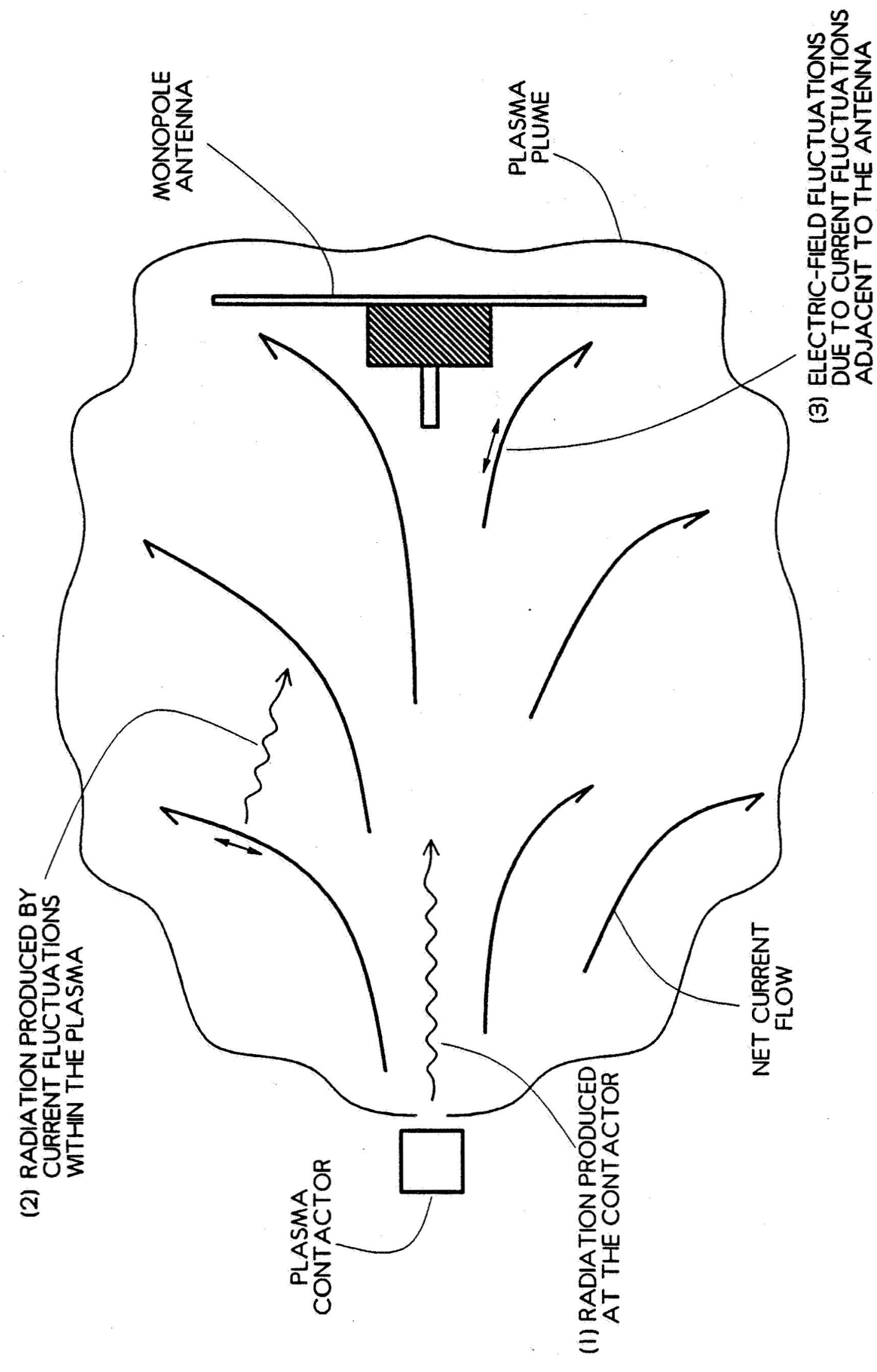

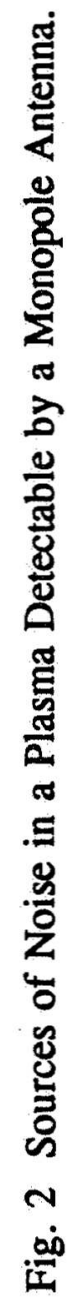




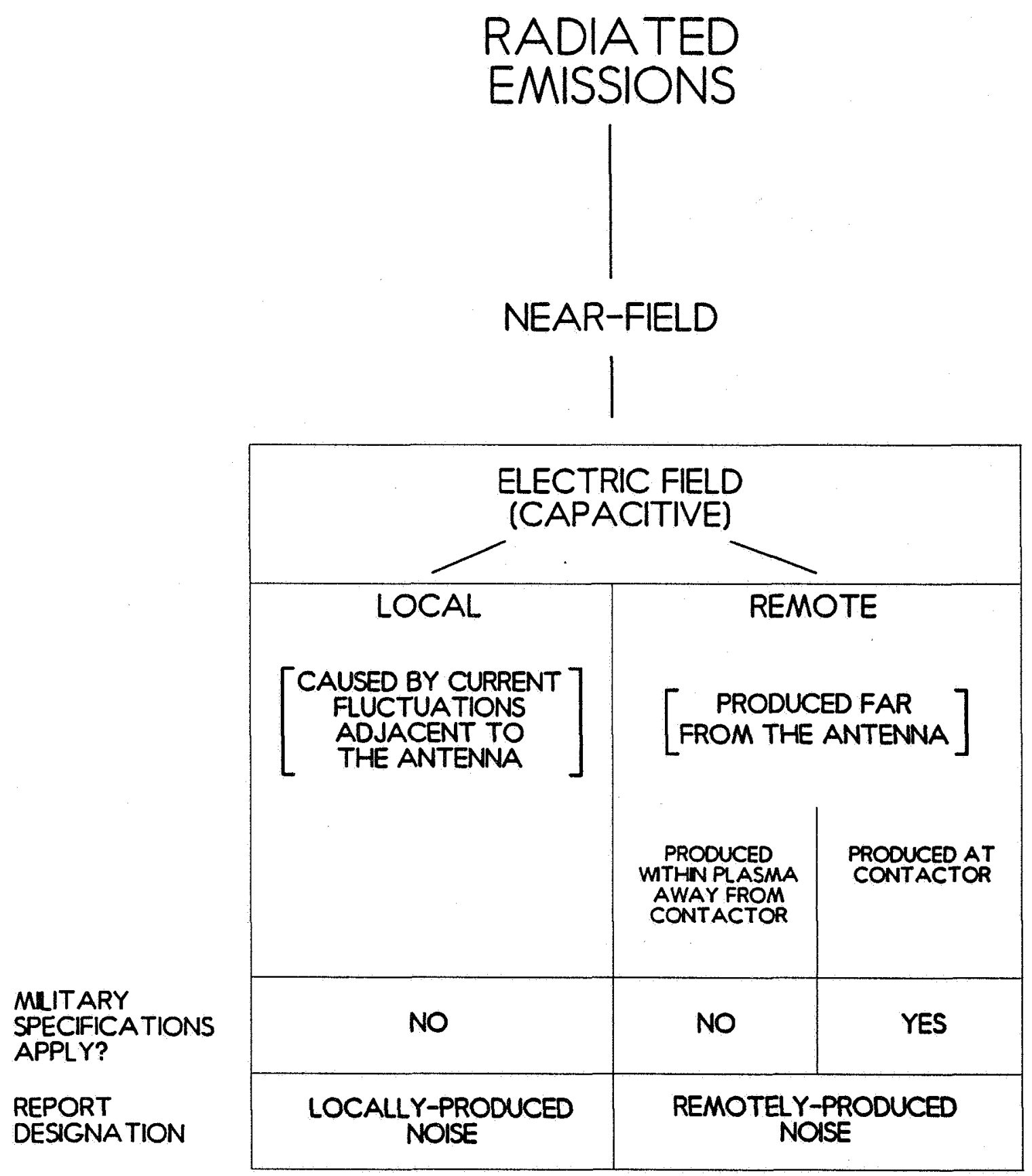

Fig. 3 Categorization of Radiated Emissions. 
Included in Fig. 3 is a description of which contactor noise production mechanisms are addressed by existing military testing standards which have been used extensively in the past to regulate RE on spacecraft [refs. 15 and 16, for example]. The locally-produced noise in particular complicates the interpretation of RE measurements in accordance with these standards. This complication is a consequence of the fact that the standards [17] and specifications [18] apply in general to "black boxes" consisting of self-contained electronic components and interconnecting cables. An assumption implicit in the application of these testing standards is that the device being tested is the source of all noise. In the case of a hollow-cathode plasma contactor this means that the testing standards for RE measurements are meant to apply only to the noise produced by the contactor itself, not to any noise that may be produced in the contactor plasma. An antenna used to sense RE, however, does not distinguish between these sources of noise. Now recall that the purpose of the specifications is to assure that noise associated with plasma contactor operation will not interfere electrically with the operation of other equipment. Thus, both locally and remotely-produced RE must be considered when defining the noise produced by the plasma contactor. In this report, the term radiated emissions will be used to refer to all of the noise detected by a monopole antenna. In addition to problems related to the sources of RE, other problems arise when RE associated with a plasma device are measured with an antenna in groundbased facilities. For example, when tests are performed in metal vacuum chambers, EM waves that are produced by contactor operation will reflect from chamber walls and create regions of constructive and destructive interference that could lead to 
inaccurate RE measurements in those regions. Whether teflections are significant or not depends on the frequency of the wave and its wavelength compared to the experimental system dimensions and this must be investigated. Furthermore, the conductive surfaces of an antenna immersed in the contactor plasma plume may interact with the plasma and affect the ability of the antenna to make accurate measurements. These problems must be overcome, or at least their effects must be recognized and taken into account, before RE can be reliably measured.

Additionally, it is desirable to determine the causes of plasma contactor RE so that noise levels may be reduced through engineering design and proper choice of operating conditions. Conducted emissions also need to be measured and their relationship to RE needs to be determined so that physical relationships between RE and $\mathrm{CE}$ can be established. Sources of noise which may be specific to ground-based tests (e.g. those due to laboratory power supplies) also need to be identified.

Experimental [19] and theoretical [20] analyses have suggested a relatively good correlation between the magnitudes and trends of RE and CE. A theoretical model [20] has been used to predict RE data using experimentally obtained CE data, and these predicted RE data match the RE measured with a monopole antenna quite well. The model suggests that at low frequencies (less than $1 \mathrm{MHz}$ ) the contactor plasma behaves as a resistive medium and that the noise sensed by the antenna at those frequencies is due to current fluctuations in the conducting plasma near the antenna rather than to remotely-produced radiation. At frequencies greater than $1 \mathrm{MHz}$, it is suggested that $\mathrm{RE}$ resulting from the time-varying current fluctuations in 
the plasma far from the antenna begin to contribute to the electric-field fluctuations sensed by the antenna.

Finally, it should be noted that plasma contactor experiments have been conducted in low-Earth orbit from the space shuttle cargo bay. During these tests, there were no reports of contactor operations interfering with either orbiter systems or other scientific payload experiments [21]. This is a good indication from space-based experiments that contactor RE and CE may be within allowable levels for spacestation operation.

This thesis investigates 1) the influence of the contactor plasma on the operation of a monopole antenna used to measure RE and methods that can be used to characterize and compensate for this, 2) difficulties associated with measuring contactor noise when operating in ground-based facilities, 3) the sources of and the relationship between $\mathrm{RE}$ and $\mathrm{CE}$, and 4) the appropriateness of testing standards and specifications derived from military ones for contactor RE measurements. 


\section{EXPERIMENTAL APPARATUS AND PROCEDURES}

Many of the experiments performed in this study were conducted in the 5.3-m long by $1.2-\mathrm{m}$ diameter stainless-steel vacuum-tank facility shown schematically in Fig. 4. The plasma contactor was situated at one end of the tank, and a simulator, that sustained an ambient plasma representative of the space plasma, was located $2.7 \mathrm{~m}$ from it. The contactor could be biased to emit electrons to the simulator and the tank walls through the ambient plasma. A monopole receiving antenna was positioned on a cart that could be traversed along the tank to measure RE at various locations. Except where specifically mentioned, the vacuum-tank RE data that will be presented in this paper were acquired with the antenna monopole tip $130 \mathrm{~cm}$ from the contactor. The contactor, simulator, and receiving antenna monopole were coaxial with the tank centerline. For certain tests, a broadcast antenna was also placed in the vacuum tank as shown in Fig. 4; it was removed when not needed for a specific experiment.

Some of the experiments were performed in the vacuum-tank facility with a different equipment configuration, namely that shown in Fig. 5. In this altered setup the locations of the contactor and simulator were interchanged and they were positioned along the tank centerline so that they were facing each other. The receiving antenna was mounted on the cart with its baseplate horizontal and the 


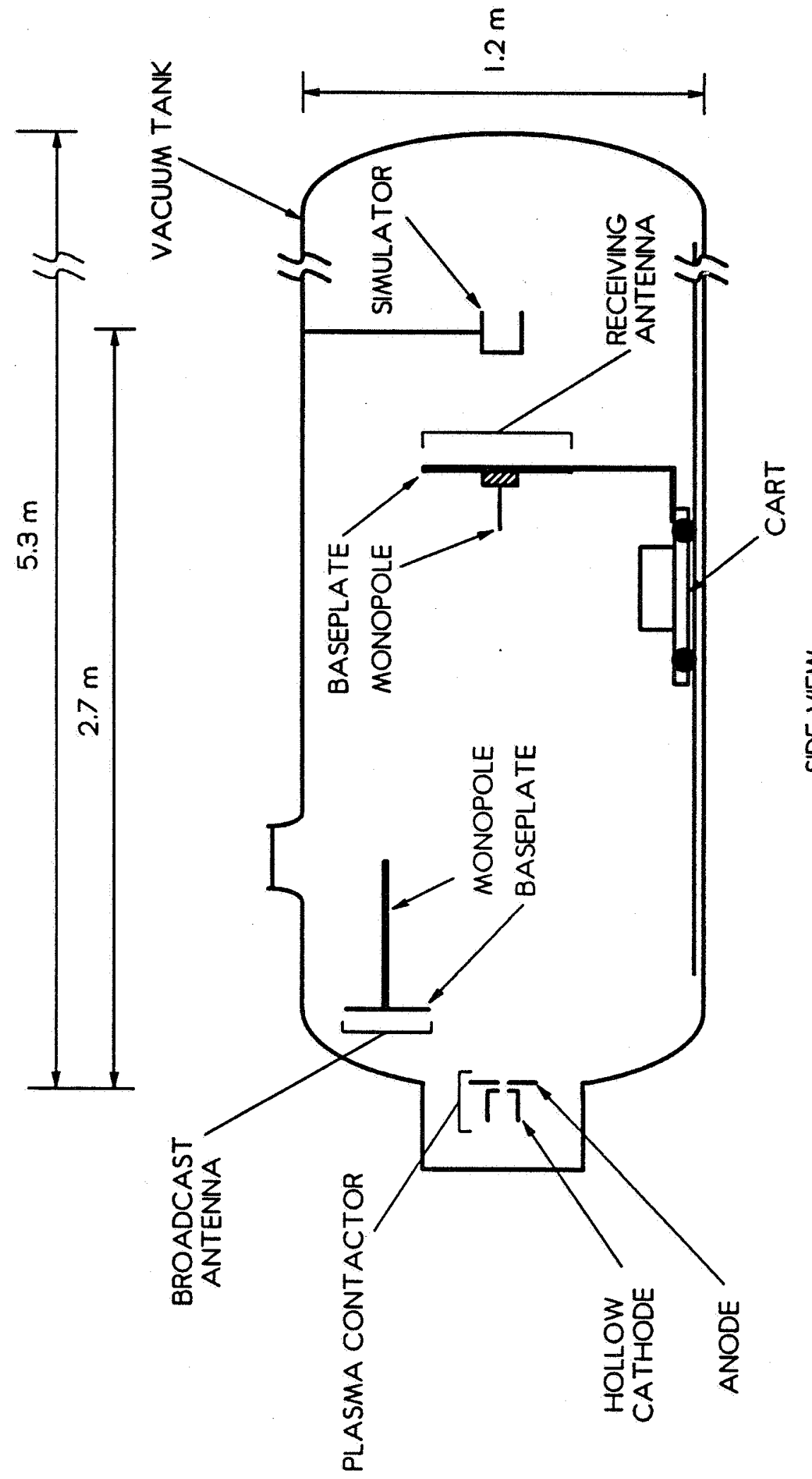

咯

总 


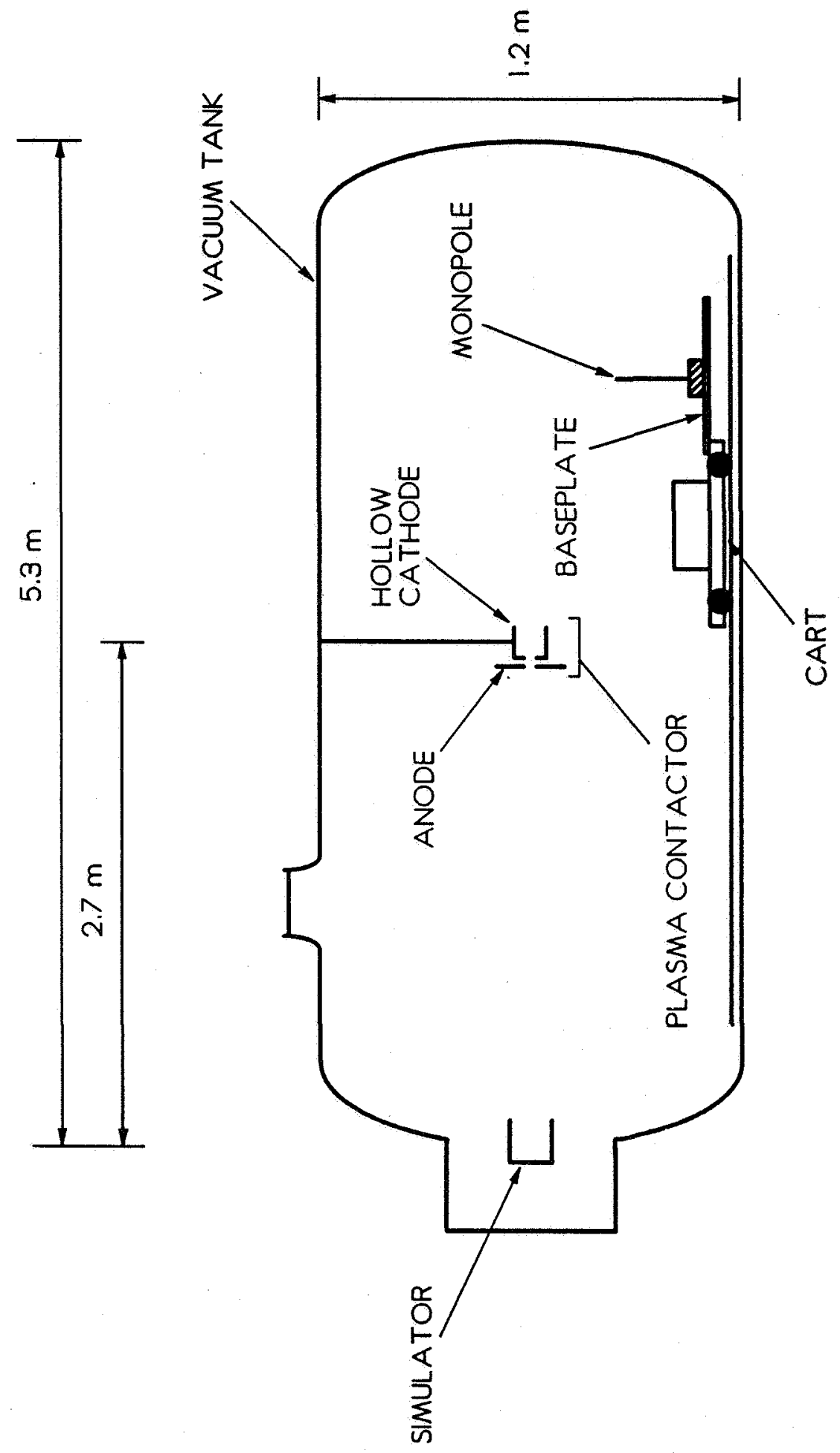

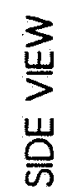

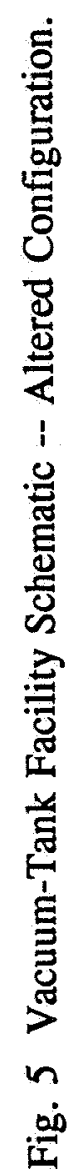


monopole pointed upward so that the antenna could be positioned behind the contactor to make noise measurements there. Experiments conducted with the altered configuration will be specifically identified in the text.

The plasma contactor used in this study, which is shown schematically in Fig. 6a, consisted of a hollow cathode, through which xenon (Xe) expellant was supplied at a rate $\dot{\mathrm{m}}_{\mathrm{c}}$, and an anode composed of a stainless-steel disc and a tantalum loop in the same plane. An electrical discharge was established between the hollow cathode and the anode, and this discharge sustained the plasma plume that contacted the ambient plasma. The cathode insert was made of tantalum foil wound as a hollow cylinder and coated with a low work-function material $\left(\mathrm{BaCO}_{3}\right.$ and $\left.\mathrm{SrCO}_{3}\right)$ which would emit electrons when heated. These electrons would ionize the expellant gas and flow to the downstream anode, simulator, and tank wall surfaces. Hollowcathode operation has been described in detail elsewhere $[1,2]$.

The simulator, shown in Fig. $6 \mathrm{~b}$, produced a low-temperature, diffuse plasma by ionizing Xe expellant in a small discharge chamber that employed a hot-filament cathode [22]. Several magnets were placed in the discharge chamber to induce a magnetic-field environment that would improve the expellant ionization efficiency by impeding the direct flow of electrons from the tungsten-wire cathode to the dischargechamber body (anode). This relatively quiescent, hot-filament-cathode device was used so noise that might be produced by a second hollow cathode in the simulator would not be present to complicate the experimental results.

The contactor, simulator and tank were connected together electrically as shown in the schematic of Fig. 7. Power supplies are depicted in the figure as 


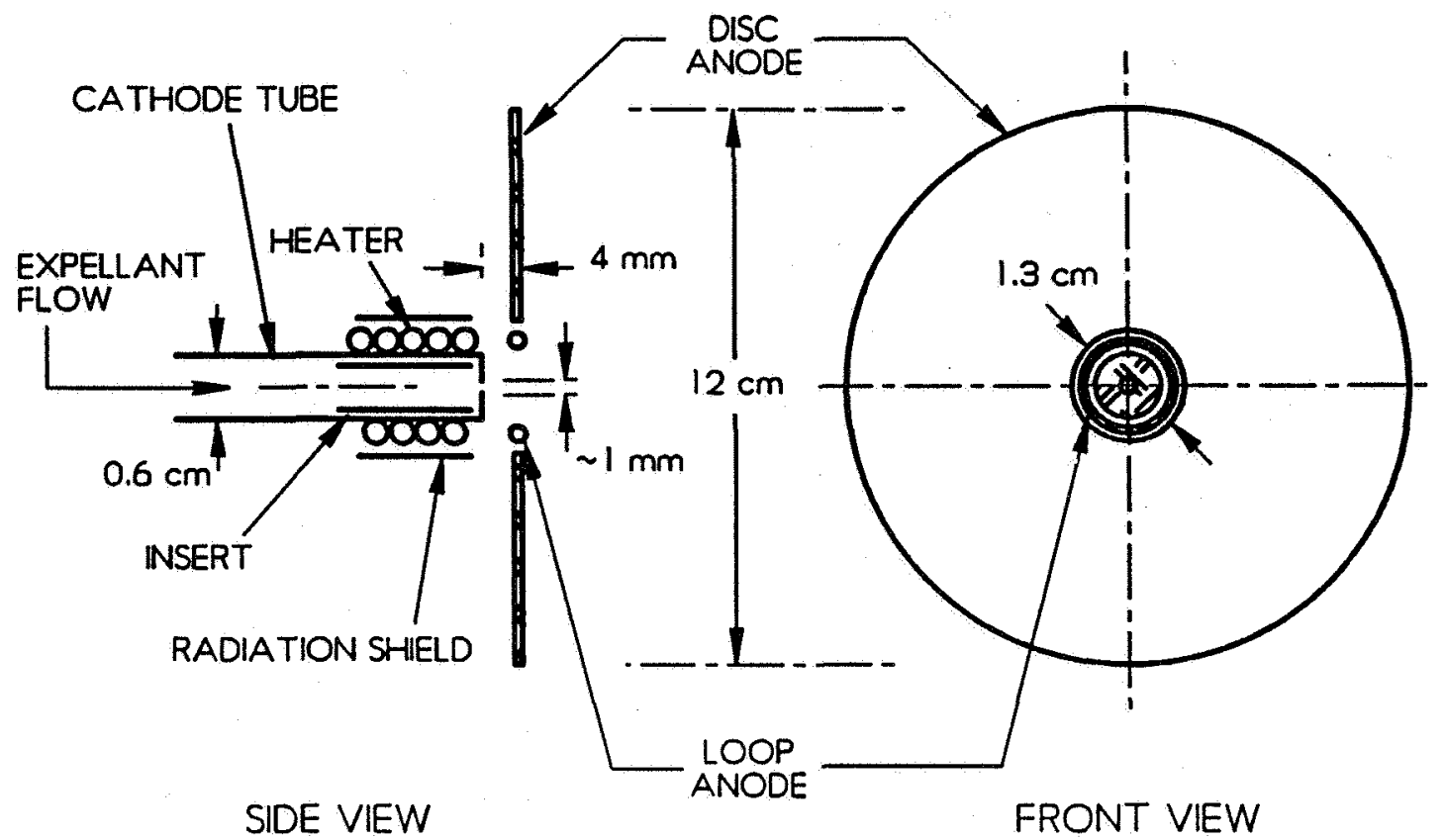

a) Hollow-Cathode Plasma Contactor Schematic.

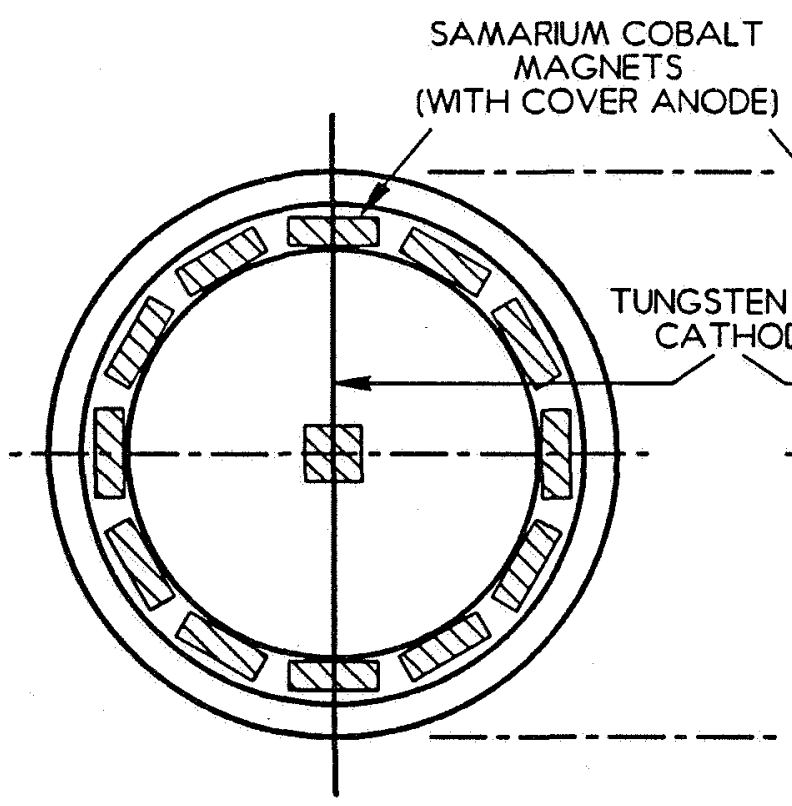

FRONT VIEW

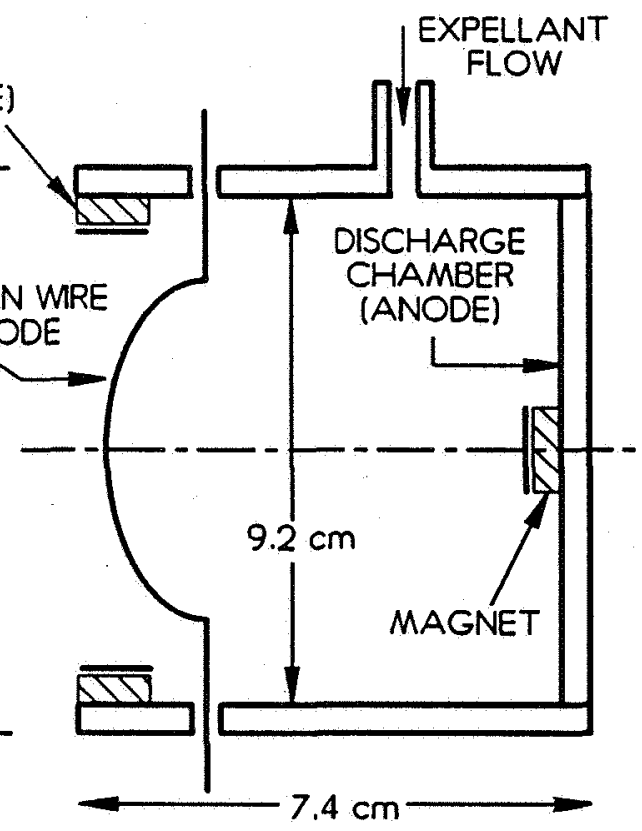

SIDE VIEW

b) Simulator Schematic.

Fig. 6 Plasma Contactor and Simulator Schematics. 


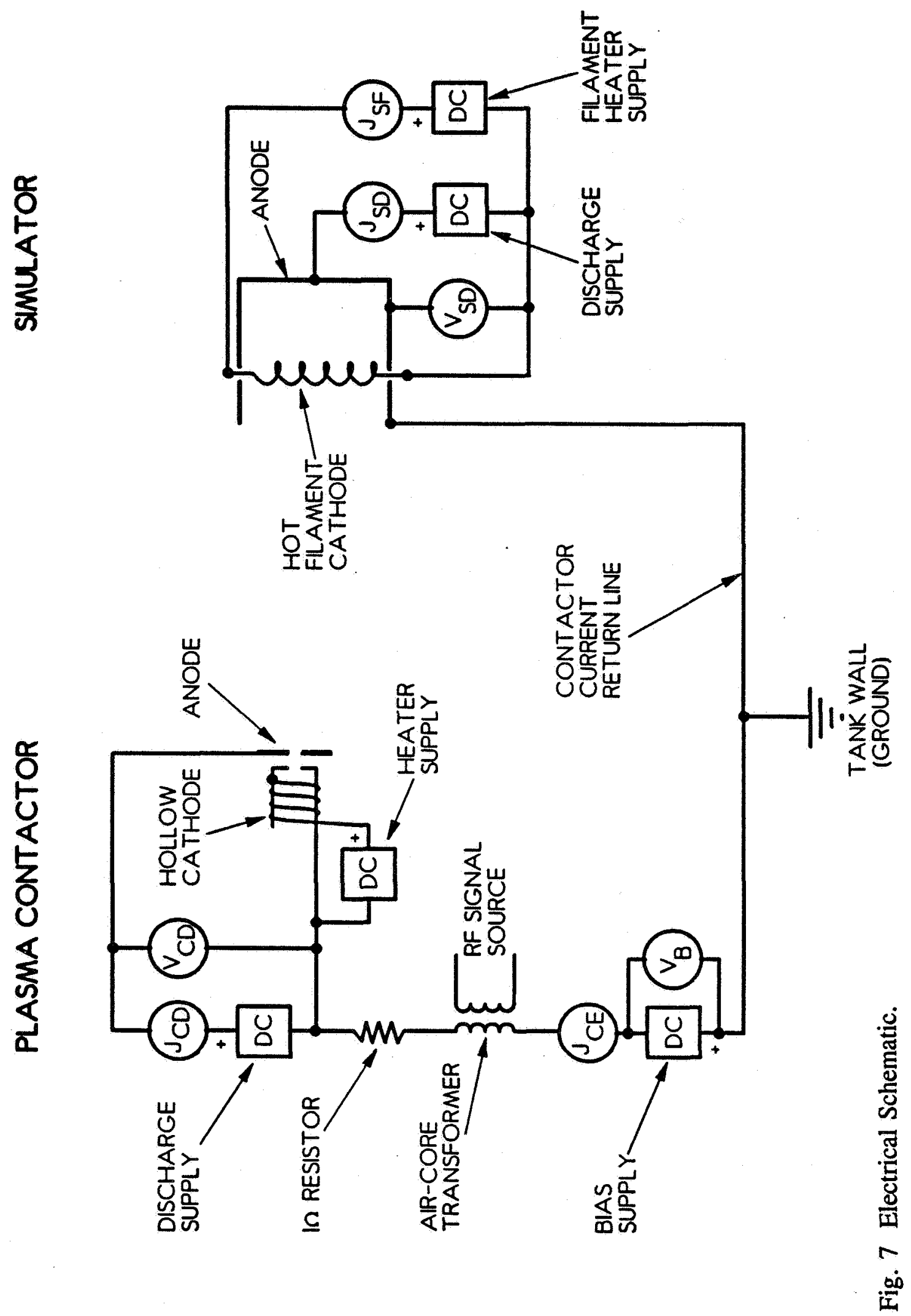


squares, and meters as circles. A discharge between the hollow cathode and anode was initiated by establishing the Xe flow, heating the cathode insert with the heater power supply and then increasing the contactor discharge voltage $\left(\mathrm{V}_{\mathrm{CD}}\right)$ until a contactor discharge current $\left(\mathrm{J}_{\mathrm{CD}}\right)$ began to flow. The simulator discharge was initiated by passing a current $\left(\mathrm{J}_{\mathrm{SF}}\right)$ through the hot filament cathode that heated it to thermionic emission temperatures, then applying the simulator discharge voltage $\left(\mathrm{V}_{\mathrm{SD}}\right)$ to establish the simulator discharge current $\left(\mathrm{J}_{\mathrm{SD}}\right)$. The contactor was biased using the bias supply to a voltage $\left(V_{B}\right)$ that was sufficiently negative with respect to the simulator anode and tank walls (which were at ground potential) to induce the desired electron emission current $\left(\mathrm{J}_{\mathrm{CE}}\right)$. The majority of the electrons that were emitted by the contactor were collected on the tank walls because of their large area compared to the simulator anode. (Although the contactor could also be biased positive of the simulator to collect electrons from the simulator, this study focuses only on electron emission).

Also shown in Fig. 7 is a $1-\Omega$ shunt resistor which was used to monitor the current fluctuations (conducted emissions) in the contactor-current return line. Lastly, the air-core transformer shown was installed so that current fluctuations could be induced at a prescribed frequency in the contactor-current return line. The transformer was driven by a radio-frequency (RF) power amplifier which received input from a signal generator.

Noise measurements were also made when the plasma contactor was operating in the bell-jar facility, shown in Fig. 8. No simulator was used in this facility; the electrons which were emitted from the contactor flowed to a thin annular electron- 


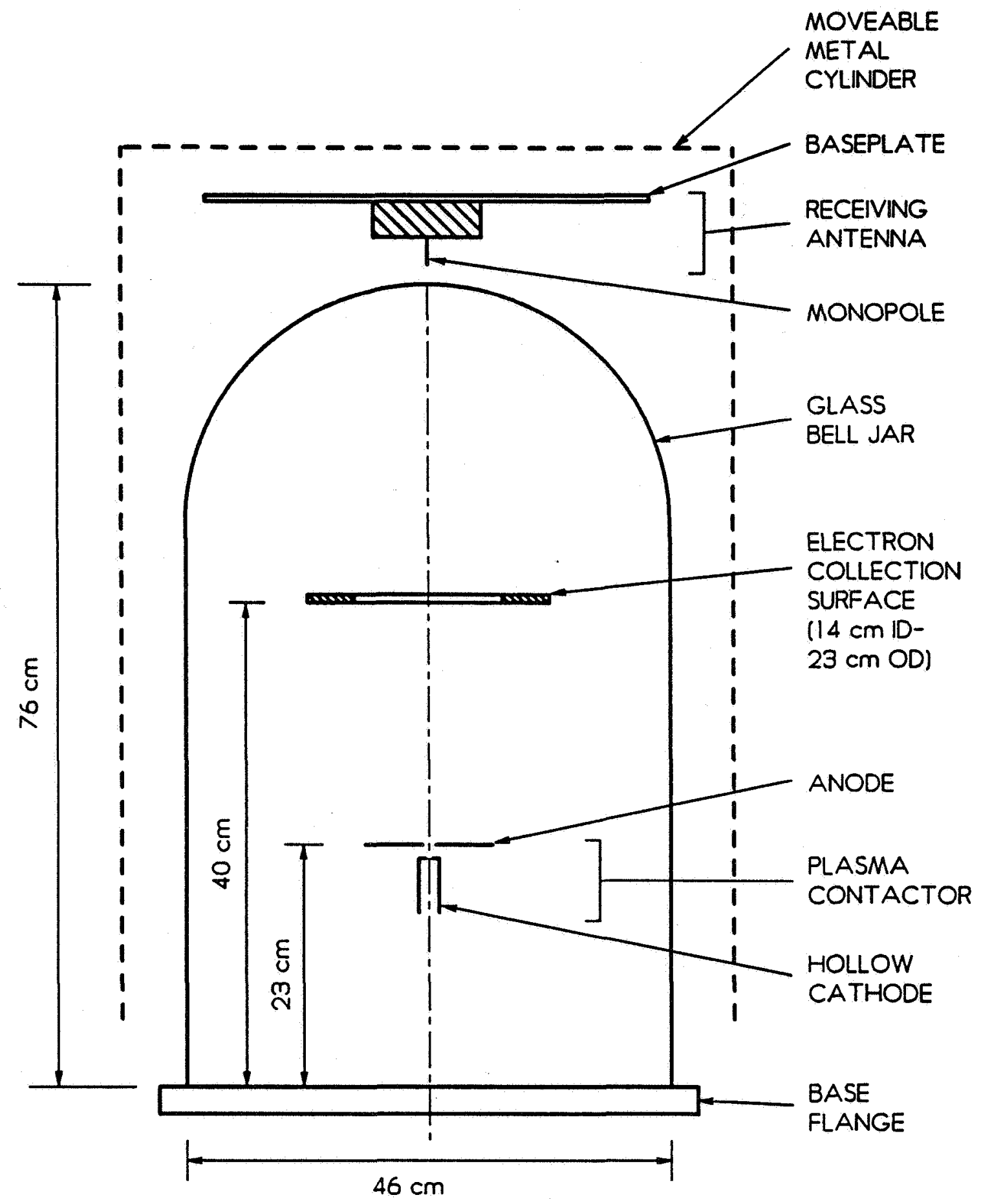

Fig. 8 Bell-Jar Facility Schematic. 
collection surface located downstream of the contactor. Experiments were conducted in the bell-jar facility because 1) RE measurements could be made with the antenna external to the system (i.e. not immersed in the plasma), and 2) the glass bell jar is a non-conducting surface and thus will not reflect EM waves.

Indicated in Fig. 8 by dashed lines is a moveable metal cylinder that could be placed over the bell jar and antenna. The metal cylinder was used to determine the changes in RE induced by placing this conducting surface around the plasma contactor. The receiving antenna used in the bell-jar tests was the same receiving antenna used in the vacuum-tank tests and it could be positioned at various locations above the contactor with its monopole aligned along the contactor centerline. The electrical circuit that was used for bell-jar experiments is the same as that shown in Fig. 7, except the simulator and its associated power supplies were replaced with the annular electron-collection surface. Additionally, the bell-jar electrical circuit was allowed to float with respect to the aluminum base flange which was at ground potential.

For experiments conducted in the vacuum tank, the standard operating conditions were a discharge current $\left(\mathrm{J}_{\mathrm{CD}}\right)$ of 0.6 Amperes and an expellant flow rate $\left(\dot{\mathrm{m}}_{\mathrm{c}}\right)$ of $4.0 \mathrm{sccm}$ of Xe. The contactor typically was operated in the 'clamped mode' $\left(\mathrm{J}_{\mathrm{CE}}=2 \mathrm{~A}\right)$ or the 'idle mode' $\left(\mathrm{J}_{\mathrm{CE}}=0 \mathrm{~A}\right)$ although other emission currents were also investigated. These standard conditions were the same for the bell-jar facility except a lower expellant flow rate $(0.72 \mathrm{sccm} X e)$ was required to assure stable operation at the desired contactor operating points. The pressure $\left(\mathrm{P}_{\mathrm{o}}\right)$ in the vacuumtank and bell-jar facilities during experiments was nominally 20-30 $\mu$ Torr. 
The monopole receiving antenna used for these experiments consisted of a $51-\mathrm{cm} \times 51-\mathrm{cm}$ baseplate, signal amplifier, and a telescoping monopole with a diameter that depended on the monopole length. The length was varied by collapsing or removing parts of the monopole to obtain lengths of $19 \mathrm{~cm}(0.7 \mathrm{~cm}$ dia. $), 1.8 \mathrm{~cm}$ (1.1 $\mathrm{cm} \mathrm{dia.)}$, or $0.5 \mathrm{~cm}(0.3 \mathrm{~cm} \mathrm{dia.)}$. Electric-field fluctuations sensed by the antenna were computed using the signal output of the amplifier and a calibration factor, provided by the manufacturer for each of the different monopole lengths used in this study. The broadcast antenna was constructed using a $41-\mathrm{cm}$ diameter baseplate and a $56-\mathrm{cm}$ long, 1.9-cm diameter monopole wrapped with a thin dielectric material and was driven by a signal generator $(5 \mathrm{~V}$ maximum peak-to-peak amplitude and 50- $\Omega$ impedance). The broadcast antenna monopole and baseplate are physically small compared to the shortest wavelength of radiation investigated in this study $(\lambda / 18$ and $\lambda / 23$, respectively). The broadcast antenna calibration factor is not known, but the frequency-dependant radiation pattern was measured as described in Appendix A.

Noise measurements were made by recording the voltage fluctuations across the shunt resistor (CE) and the signal output from the receiving antenna amplifier (RE) on an oscilloscope. Typically, these data were acquired sequentially although they were occasionally acquired simultaneously to demonstrate time correlation between signals. The frequency ranges and resolution bandwidths with which data were recorded, explained in ref. 15 , are dictated by established testing standards. The data, which then were analyzed on a computer using a Fast-Fourier-Transform technique [19], are presented in two different ways. Radiated emissions are displayed in the power spectral density (PSD) format, i.e. mean-squared signal amplitude 
divided by the resolution bandwidth, in the units used in typical NASA specifications $\left(\mathrm{dB}[\mu \mathrm{V} / \mathrm{m} / \mathrm{MHz}] \equiv 10 \log _{10}\left[(\mu \mathrm{V} / \mathrm{m})^{2} / \mathrm{MHz}\right]\right)[15,16]$. Conducted emissions are shown as current fluctuation amplitude divided by emission current $(\mathrm{dB}[\mu \mathrm{A} / \mathrm{A}] \equiv$ $\left.20 \log _{10}[\mu \mathrm{A} / \mathrm{A}]\right)$. In some cases, $\mathrm{RE}$ and $\mathrm{CE}$ data are both presented together in the PSD format $\left(\log _{10}\left[(\mathrm{~V} / \mathrm{m})^{2} / \mathrm{Hz}\right]\right.$ and $\log _{10}\left[\mathrm{~mA}^{2} / \mathrm{Hz}\right]$, respectively) which facilitates the identification of frequency-dependent noise structures and also direct comparison of corresponding RE and CE data.

The noise data acquired during this experimental investigation were found to be fairly repeatable within each experiment and also from day-to-day. Typical RE data acquired with the monopole antenna when the plasma contactor was operating in the clamped mode are plotted in Fig. 9 as $\mathrm{dB}(\mu \mathrm{V} / \mathrm{m} / \mathrm{MHz})$ v. frequency for experiments performed on two different days (Tests A and B). It can be seen that the two sets of data from Test A are very similar. At the majority of frequencies they agree to within $1-2 \mathrm{~dB}$, although there are rare differences of as much as $10 \mathrm{~dB}$ near $30 \mathrm{kHz}$ and $3 \mathrm{MHz}$. The measurement uncertainty due to the data acquisition and analysis process is on the order of $2 \mathrm{~dB}$ [19] so these data are considered to be essentially identical. Comparison of the RE data from Tests A and B demonstrates that the day-to-day measurement repeatability is also fairly good. Differences between RE data are less than about $10 \mathrm{~dB}$ at all frequencies. Analysis of CE data showed repeatability similar to that of the RE data. Thus, when comparing noise data acquired during this study, differences of $10 \mathrm{~dB}$ or less will generally be considered to be within day-to-day repeatability. 


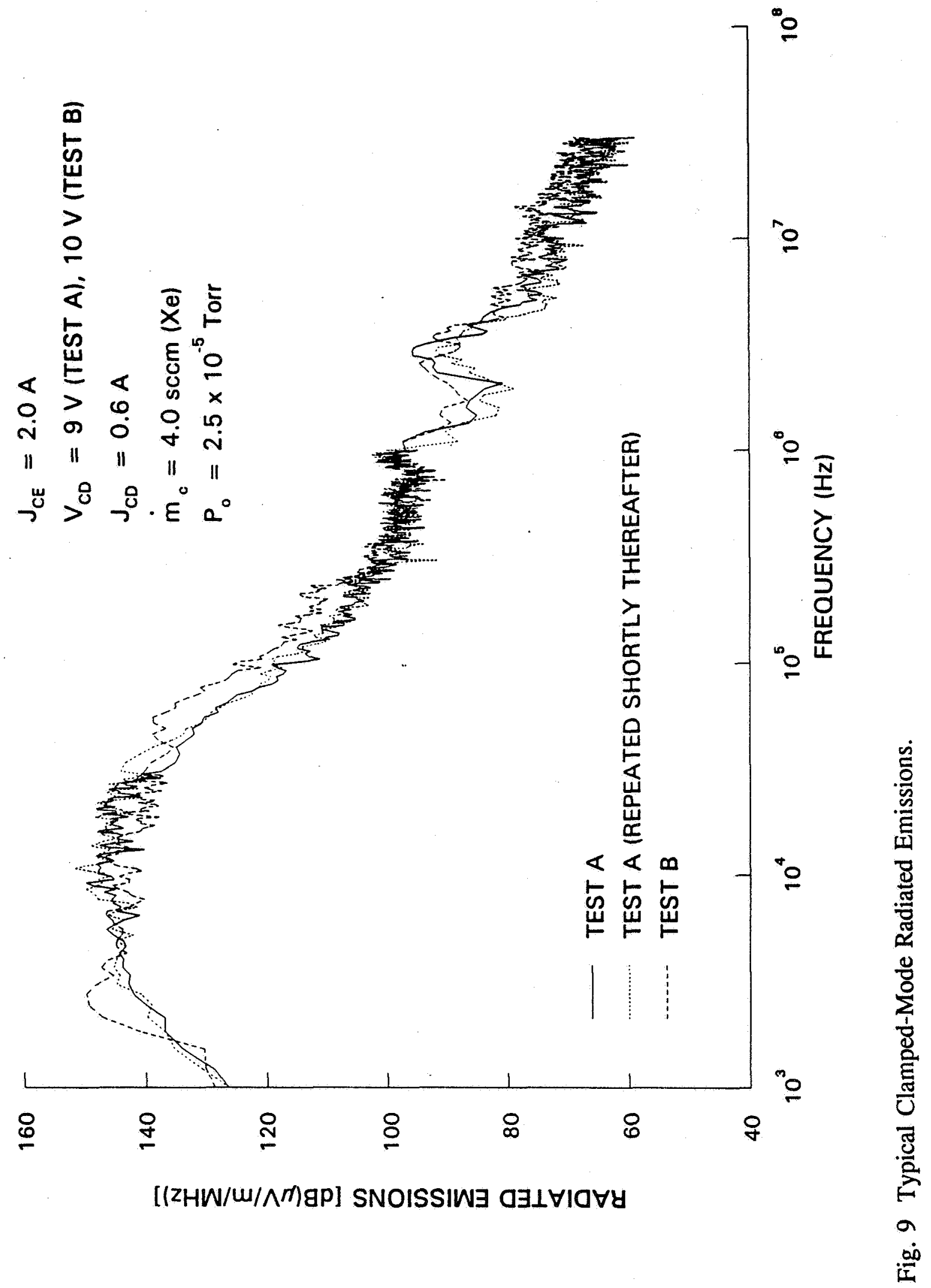


The RE data that are presented in this report will also be compared to specifications that have been set for the maximum allowable RE produced by the plasma contactor. The specifications shown will be those set by the U.S. military for broadband RE produced by equipment installed on spacecraft $[17,18]$. Recall, however, that caution must be exercised when comparing RE data to specifications because of the inherent assumption in the military testing standards that noise is generated at the device under test only. 


\section{EXPERIMENTAL RESULTS AND DISCUSSION}

\section{Antenna Measurement Methods in a Plasma}

It has been shown that signal levels measured using a monopole antenna with its conductive surfaces exposed to a plasma can be reduced substantially below true values because of signal current flow through a low-impedance path which can develop through the plasma between the monopole and baseplate [19]. Those experiments were performed with the antenna baseplate fixed at ground potential. In this study, a series of experiments was conducted to investigate the interaction of the ambient plasma with the conductive surfaces of the antenna when the baseplate potential was changed. Specifically, it was desired to determine which antenna configuration, i.e. the amount of isolation from the plasma and also the potential of the baseplate, would yield the most accurate measurements.

An initial test was conducted in the vacuum-tank facility in which RE were measured with the monopole and baseplate exposed to the plasma and with the baseplate either floating or grounded (i.e. electrically connected to the tank wall which was collecting most of the electrons emitted by the contactor). The results of this test are shown in Fig. 10 where RE data are plotted as $d B(\mu \mathrm{V} / \mathrm{m} / \mathrm{MHz}) \mathrm{v}$. frequency. The data indicate that signals sensed by the antenna when the baseplate is grounded are substantially lower than those sensed when it is floating. In fact, the 


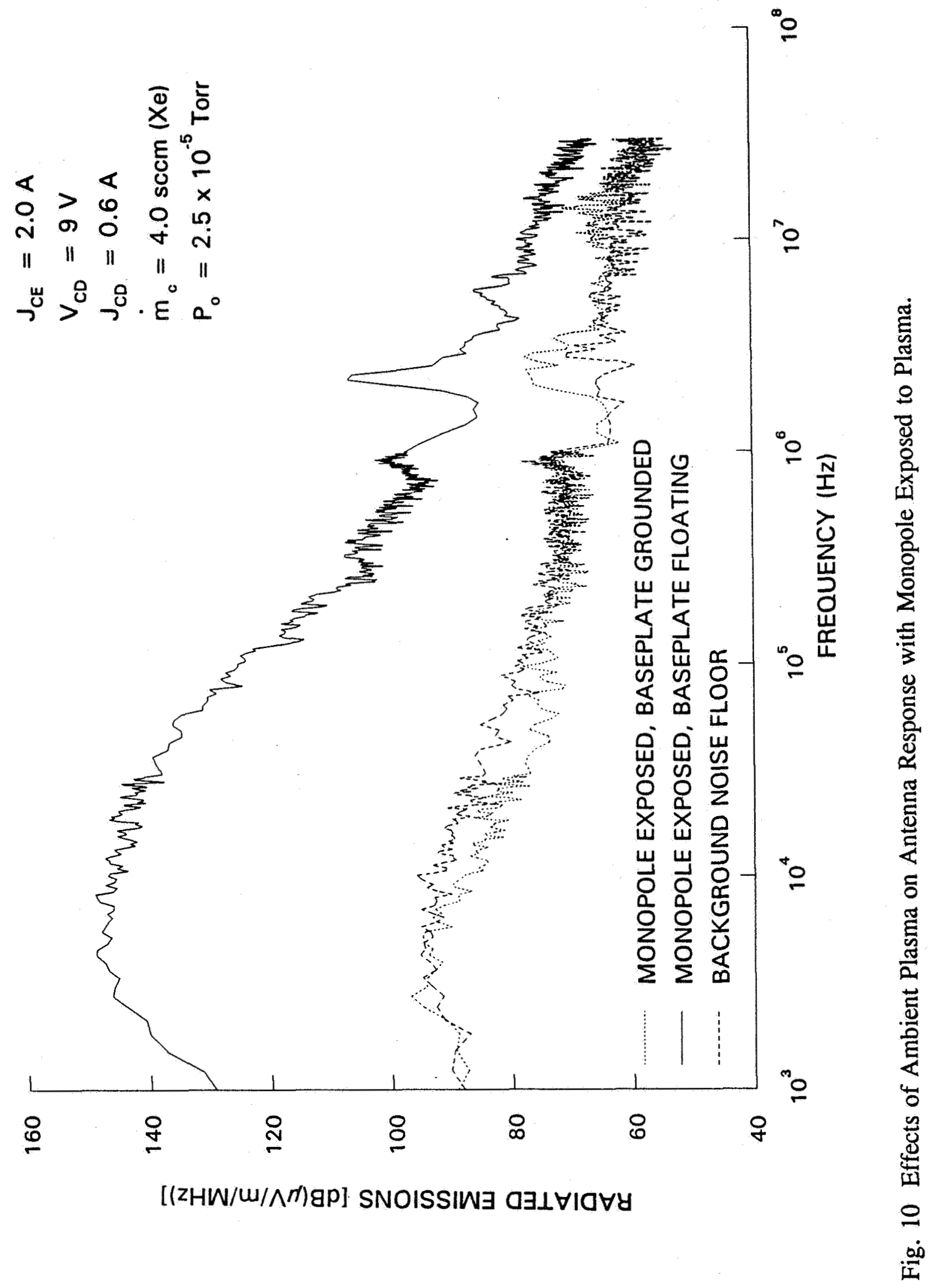


signals measured when the baseplate was grounded are nearly the same as the ambient noise floor, i.e. the signal levels measured when the plasma contactor and simulator were not operating. It appears that grounding the baseplate introduces an interference that makes the antenna insensitive.

This test was repeated in a separate experiment in which the antenna monopole was isolated from the plasma by wrapping it with a thin layer of dielectric material. Figure 11 displays the results of this test along with the RE data from Fig. 10 where the monopole was exposed to the plasma and the baseplate was floating. It is evident from the data in Fig. 11 that, when the monopole is isolated from the plasma, similar $\mathrm{RE}$ are obtained whether the antenna baseplate is grounded or floating. These data are also very similar to those acquired when the monopole is exposed to the plasma and the baseplate is floating.

The results of Figs. 10 and 11 are consistent with the results of a previous investigation [19]. It appears that, when the monopole is exposed to the plasma and the baseplate is grounded (i.e. electrically connected to the tank walls), a lowimpedance path develops between the monopole and the baseplate which substantially reduces the signal levels measured by the antenna. It was also discovered, however, that this effect was not present when the baseplate was floating instead of grounded. This suggests that, under these prevailing ambient plasma conditions, the baseplate does not have sufficient area to make direct coupling between the monopole and the baseplate significant, and that the added area of the tank walls is necessary for substantial signal reduction. 


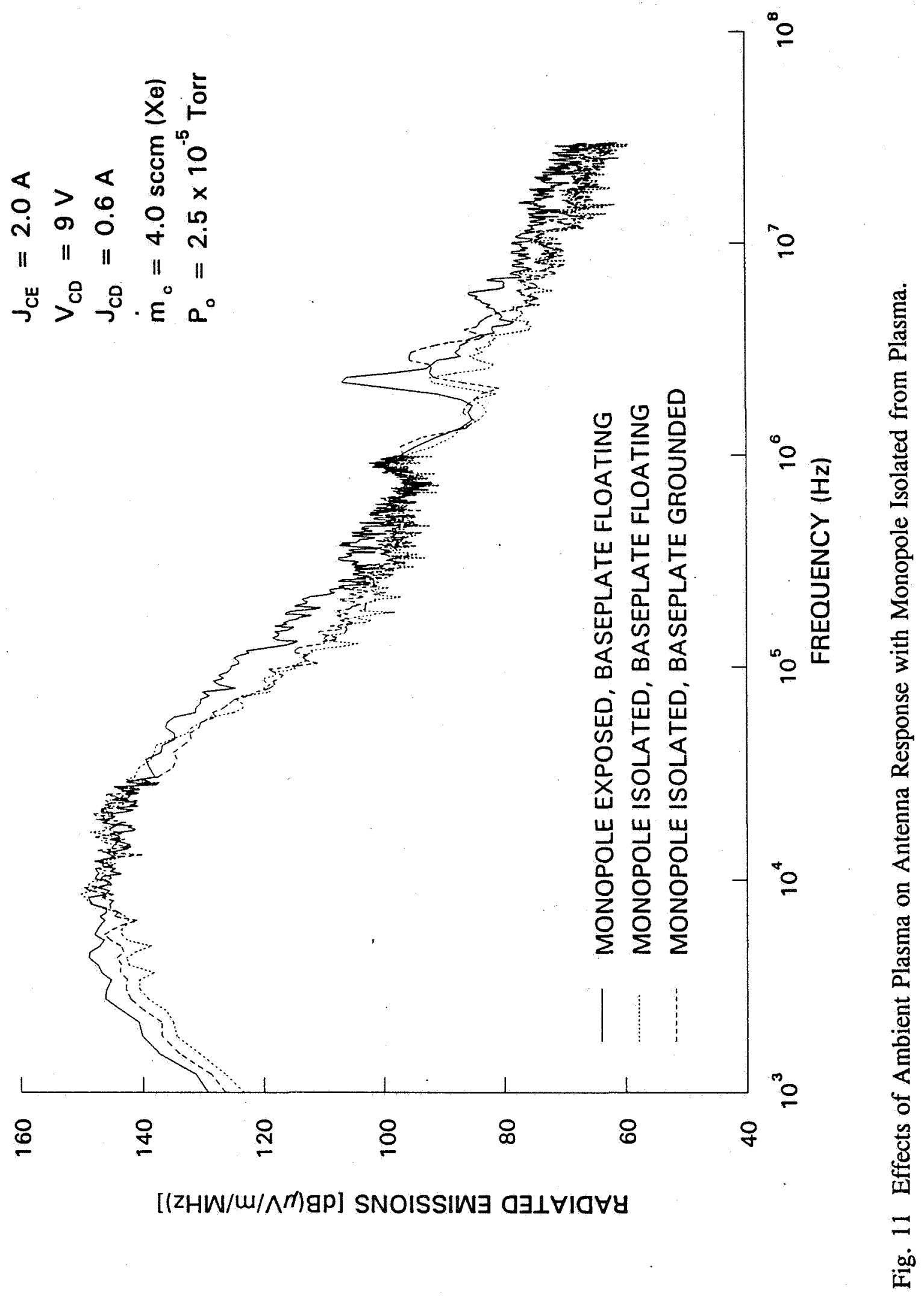


An additional experiment was conducted in which the antenna baseplate was isolated from the plasma by enclosing it in mylar. This experiment was performed when the contactor and simulator positions in the vacuum-tank were interchanged, thus the RE may be expected to be somewhat different than those shown in the previous two figures. During this test, the antenna monopole was isolated from the plasma and the baseplate was allowed to float. Radiated-emissions data acquired during this test are compared in Fig. 12 to RE data acquired in a separate test when the baseplate was exposed to the plasma. The small differences between the two RE spectra are within day-to-day variations in experimental conditions, thus it appears that isolating the baseplate from the plasma with the monopole isolated does not significantly affect antenna measurements.

The results of these experiments suggest that all that is required to reduce interference from the plasma to an acceptable level when measuring RE is to prevent the monopole from coupling to the baseplate through the tank walls. This can be accomplished by isolating the monopole from the plasma or by leaving the monopole exposed and allowing the baseplate potential to float. There is a complication associated with floating the baseplate, however, that may arise when making RE measurements with the antenna. This can be understood by considering the mechanism by which the antenna detects electric-field fluctuations. Electric-field fluctuations near the antenna induce currents on the surface of the monopole which induce a voltage difference between the monopole and baseplate across an impedance in the amplifier. The amplified voltage is the output signal from the antenna. If the baseplate is allowed to float, its potential may fluctuate in response to fluctuations in 


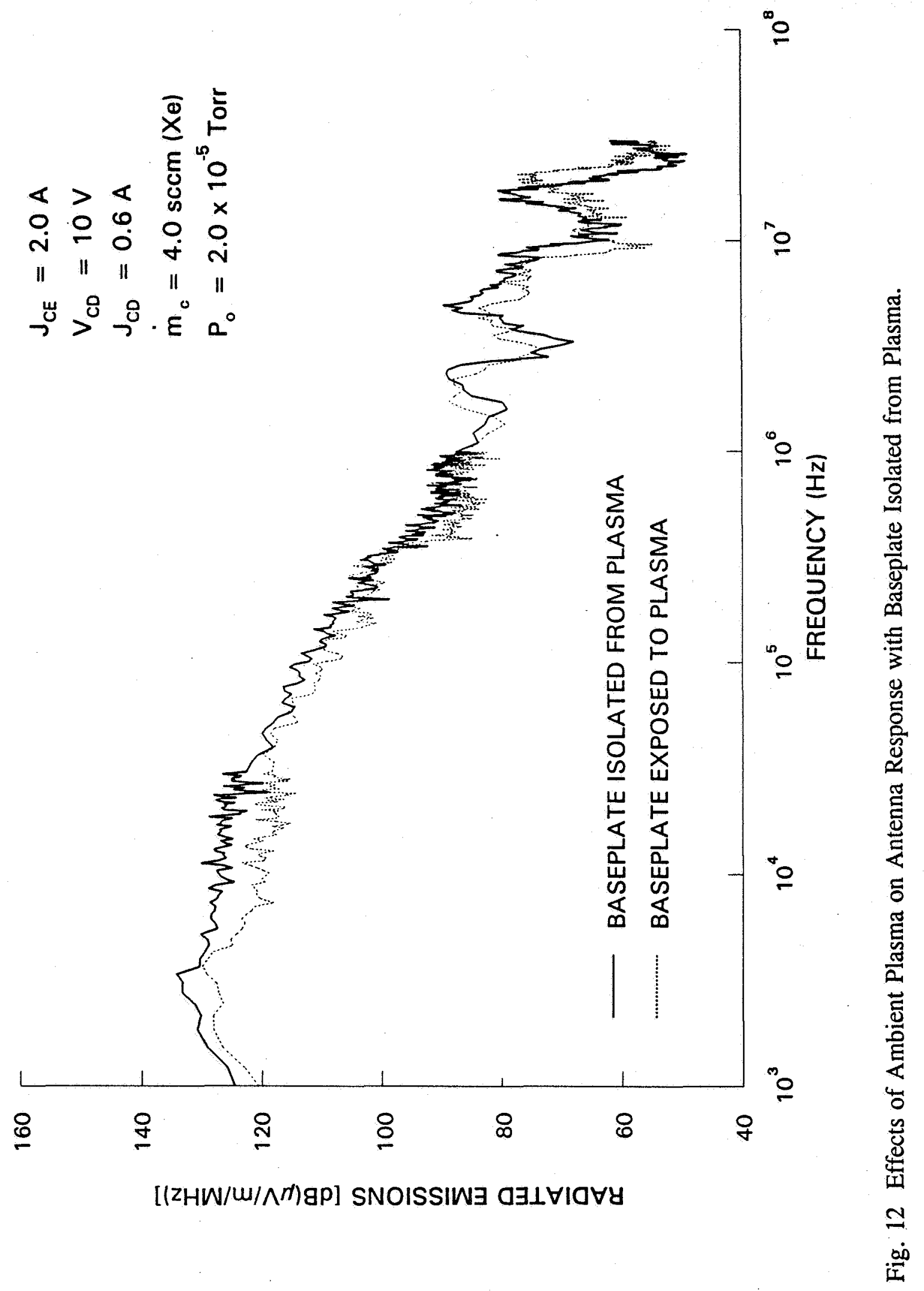


potential or density of the plasma adjacent to it. A fluctuating baseplate potential would establish a fluctuating reference potential and, if those fluctuations are large enough, would create false signals in the RE spectra. This effect may in fact be the cause of the differences observed in Fig. 11 near 2-3 MHz. Thus, it was determined that the most accurate RE measurements would be made with the antenna monopole isolated from the plasma and the baseplate grounded. Although it was also determined that the baseplate could be isolated or left exposed the plasma, it was left exposed for simplicity.

Another possible error that must be avoided when making antenna measurements in a plasma environment is saturation of the antenna amplifier circuitry. Saturation occurs when the amount of current induced in the antenna monopole by external electric-field fluctuations is too great for the amplifier circuitry to process. In such a case, the output signal is reduced in magnitude (i.e. compressed) by the circuitry. Compression can frequently be recognized in analyzed data because signal amplitudes are compressed by approximately the same amount at the majority of the frequencies investigated. Saturation and the resulting data compression may be eliminated by reducing the amount of current induced in the monopole by reducing its length. It may be concluded that the length has been reduced sufficiently when decreases in length followed by signal analysis using proper calibration factors for each length yield RE spectra that are essentially the same over the full frequency range.

Several tests were conducted to determine the monopole length that should be used to avoid amplifier saturation when RE measurements are being made. These 
tests were performed in the vacuum-tank facility with the altered configuration (Fig. 5) and the monopole $55 \mathrm{~cm}$ upstream of (behind) the contactor orifice. Figure 13 compares RE data acquired with three different monopole lengths under similar test conditions. It can be seen from the figure that the data acquired with the 73-cm-long monopole are more than an order of magnitude less than the data acquired with the other two monopole lengths at almost all frequencies; thus data acquired with the $73-\mathrm{cm}$ monopole are compressed. The data acquired with the $19-$ and $0.5-\mathrm{cm}-$ long monopoles, however, are very similar at frequencies above $20 \mathrm{kHz}$ and hence it is argued that neither of these sets of data are being compressed. At frequencies below $20 \mathrm{kHz}$ the shorter monopole gives lower output because the sensitivity of this antenna/monopole configuration is poorer at lower frequencies. Additionally, the signal-to-noise ratio in the amplifier circuitry becomes a performance-limiting factor with shorter monopoles. In fact, experiments have shown that RE data acquired with the shorter monopole $(0.5 \mathrm{~cm})$ can yield electric-field data that are masked by the noise floor in the amplifier circuitry at frequencies greater than $10 \mathrm{MHz}$, though this effect is not observed in Fig. 13. Thus, true contactor RE spectra at higher frequencies may be less than those indicated by antenna measurements made with the shorter monopoles. For these reasons, it is desirable to use the longest monopole that does not lead to saturation.

A comparison of the data in Figs. 11 and 12 shows that the magnitudes of the RE levels are generally lower in Fig. 12 than they are in Fig. 11. This is because the receiving antenna was positioned in different locations with different orientations in each experimental configuration (the reason for these different RE magnitudes will 


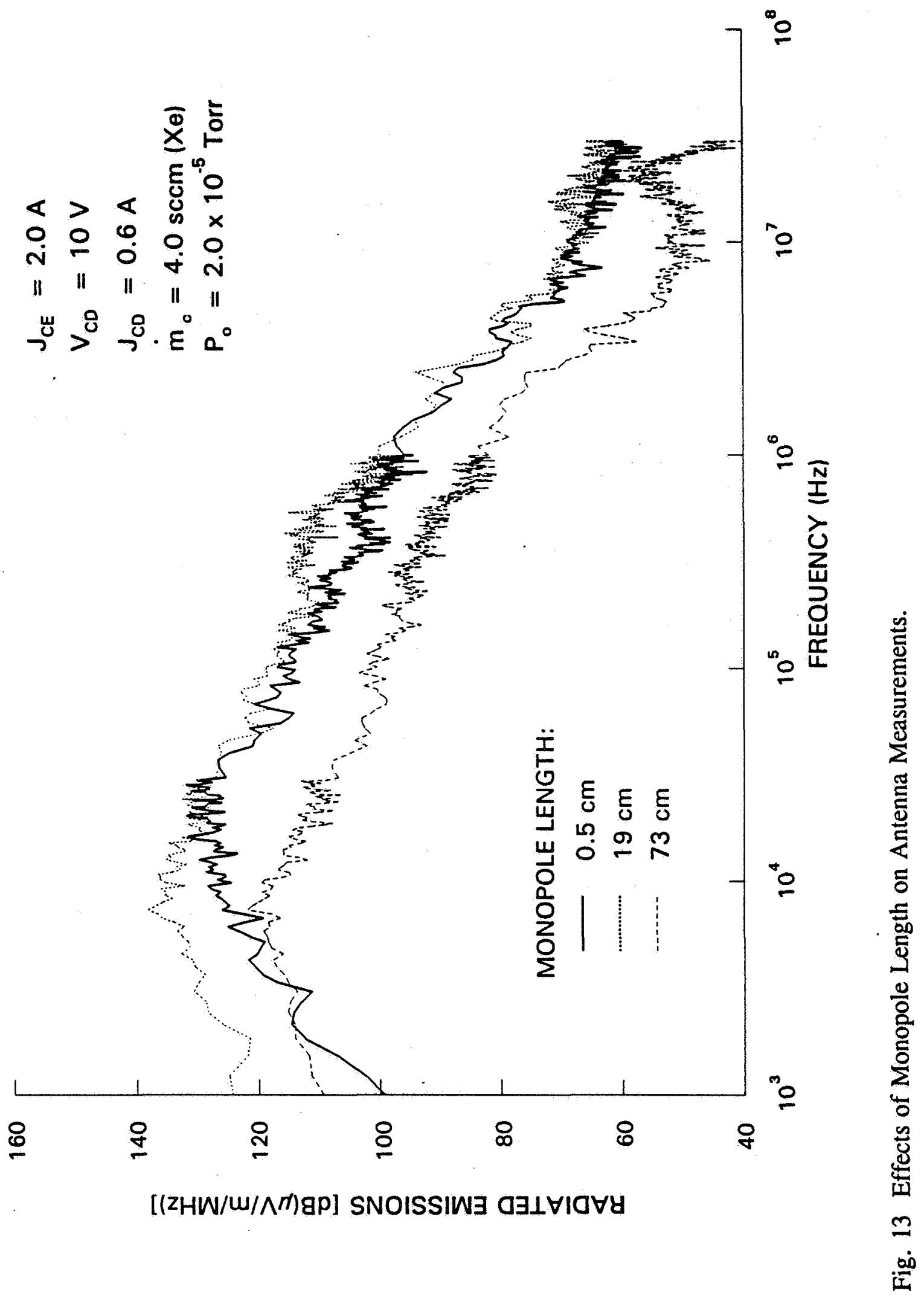


become clear later in the text). The data of Figs. 10,11, and 12 were acquired with sufficiently short antenna monopoles, and thus were not compressed.

\section{Influence of Vacuum-Chamber Walls on RE Measurements}

Several tests were conducted to determine the effects of EM wave interactions with the walls inside the vacuum tank as a function of frequency. These tests were performed without a plasma present in the tank, i.e. the contactor and simulator were not operating. The first test was conducted with the broadcast antenna monopole aligned along the tank centerline. Using the cart, the receiving antenna was moved along the tank centerline and the amplitudes of the signals from it were recorded from the oscilloscope.

Representative data for several frequencies are shown in Fig. 14 as normalized amplitude (amplitude divided by maximum amplitude) v. distance between antennae base plates. At frequencies of $1 \mathrm{MHz}$ and below, the signal levels show the same behavior along the tank centerline. At the higher frequencies, the data show markedly different behavior. This test was repeated with the broadcast antenna positioned off of the tank centerline, the position seen in Fig. 4, and these results are shown in Fig. 15. Again, at frequencies of $1 \mathrm{MHz}$ and below, the signal levels show the same behavior along the tank centerline, and at higher frequencies there is much different behavior. Although it is possible that the effects observed in Figs. 14 and 15 are due in some part to the frequency-dependent radiation pattern of the broadcast antenna, this is not considered to be a significant factor. The measured radiation 


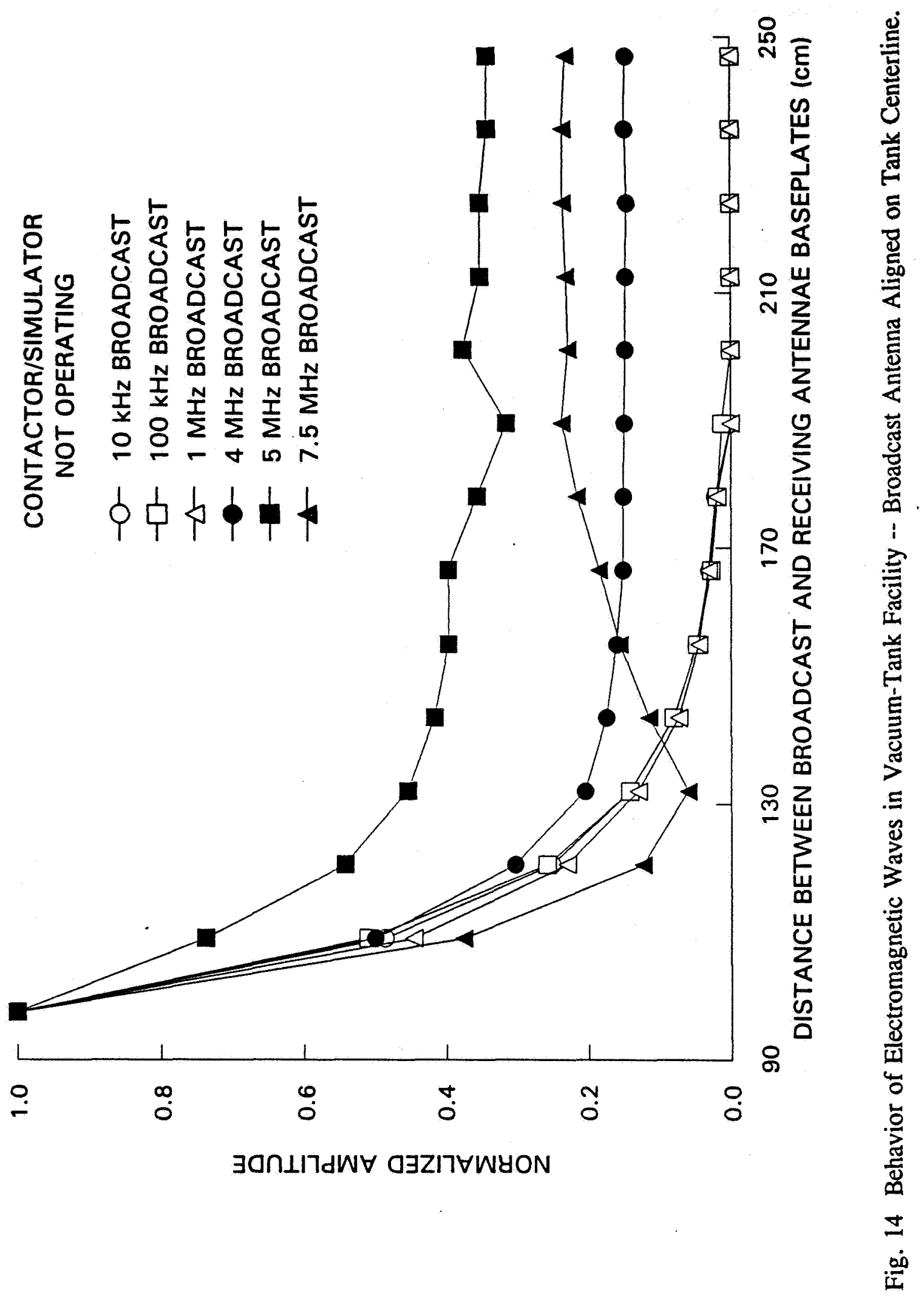




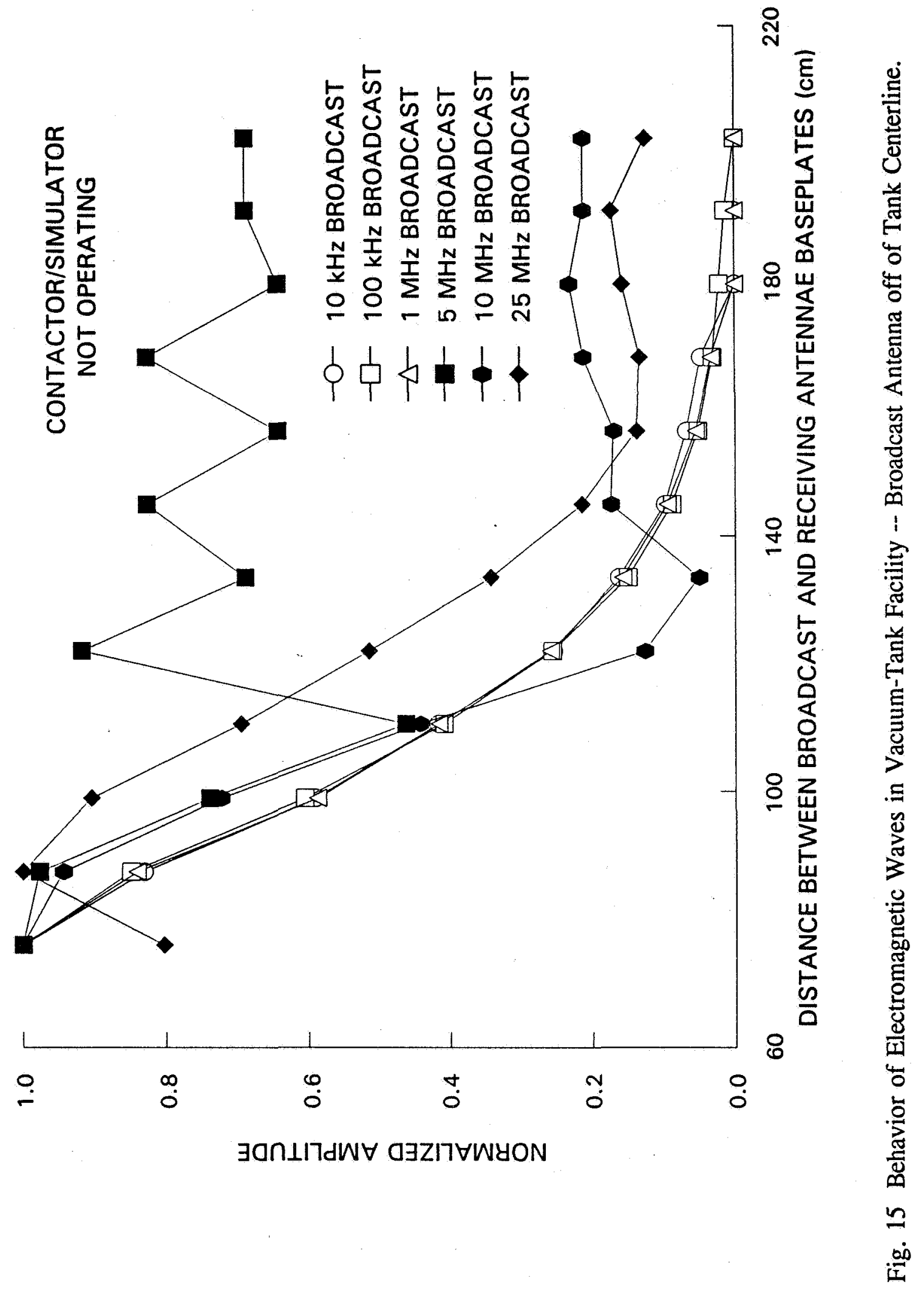


patterns for several frequencies (see Appendix A) have no prominent radiation lobes that might cause the effects seen in those figures.

The data of Figs. 14 and 15 show behavior that is indicative of that found in microwave cavity resonators. In order to determine if resonance was a possibility, the resonant electromagnetic modes of a cylindrical cavity having the same dimensions as the vacuum tank were investigated. A simple analysis [23] indicated that the lowest resonant frequency possible for the Transverse Electric mode was about $150 \mathrm{MHz}$, and that for the Transverse Magnetic mode was about $190 \mathrm{MHz}$. It is known, however, that discontinuities and obstructions in a cylindrical cavity, such as all of the equipment inside the vacuum tank, can change the resonant frequencies of the system [23]. It is unlikely, though, that this effect will cause the resonant frequencies to decrease to as low as $5 \mathrm{MHz}$, where the resonant-like behavior is seen in the vacuum tank.

Nonetheless, it is apparent from the data in Figs. 14 and 15 that some type of interaction is taking place between the vacuum-tank equipment and the EM waves that depends on frequency and location in the tank. The vacuum-tank contains many different metallic objects of various sizes including the cart and positioning system for the receiving antenna, the simulator and its associated hardware, and numerous other wires and objects that are not a part of this experimental study. The electromagnetic fields produced by the broadcast antenna will interact with all of these objects differently at different frequencies and this might affect the measurements made with the receiving antenna. Hence, it is extremely difficult to determine or predict 
accurately the extent to which the metallic surfaces in the vacuum tank will influence the measurement of plasma contactor RE with the receiving antenna.

So, while the data of Figs. 14 and 15 do not conclusively show that EM waves are being reflected from the walls of the vacuum tank, it is acknowledged that this is a possibility. Additionally, it is recognized that some phenomenon exists which causes $\mathrm{EM}$ waves at frequencies above a few $\mathrm{MHz}$ to behave unusually inside of the vacuum tank, depending on frequency and location in the tank, and that this must be remembered when interpreting RE measurements. When the plasma contactor is operating, however, the plasma in the tank will alter the behavior of the electromagnetic fields and it will also provide a mechanism for attenuation of any EM waves which are travelling through the plasma. Thus, the effects of interactions of EM waves with the metallic surfaces in the vacuum tank may be mitigated and may not even be a problem at all.

A direct comparison of EM-wave-reflection effects with and without an electrically conductive surface surrounding an operating plasma contactor was performed in the bell-jar facility. In this experiment the receiving antenna was located at the top of the glass bell jar so that the metal cylinder could be placed over both it and the bell jar easily and quickly. Figure 16 shows a typical direct comparison of clamped-mode RE data acquired with and without the cylinder surrounding the bell jar. The data indicate that, within experimental error, there is no difference in RE spectra acquired in the bell-jar facility if the plasma plume is surrounded by a conductive surface or not. Idle-mode RE data acquired with and without the metal cylinder in place are compared in Fig. 17. With the exception of a 

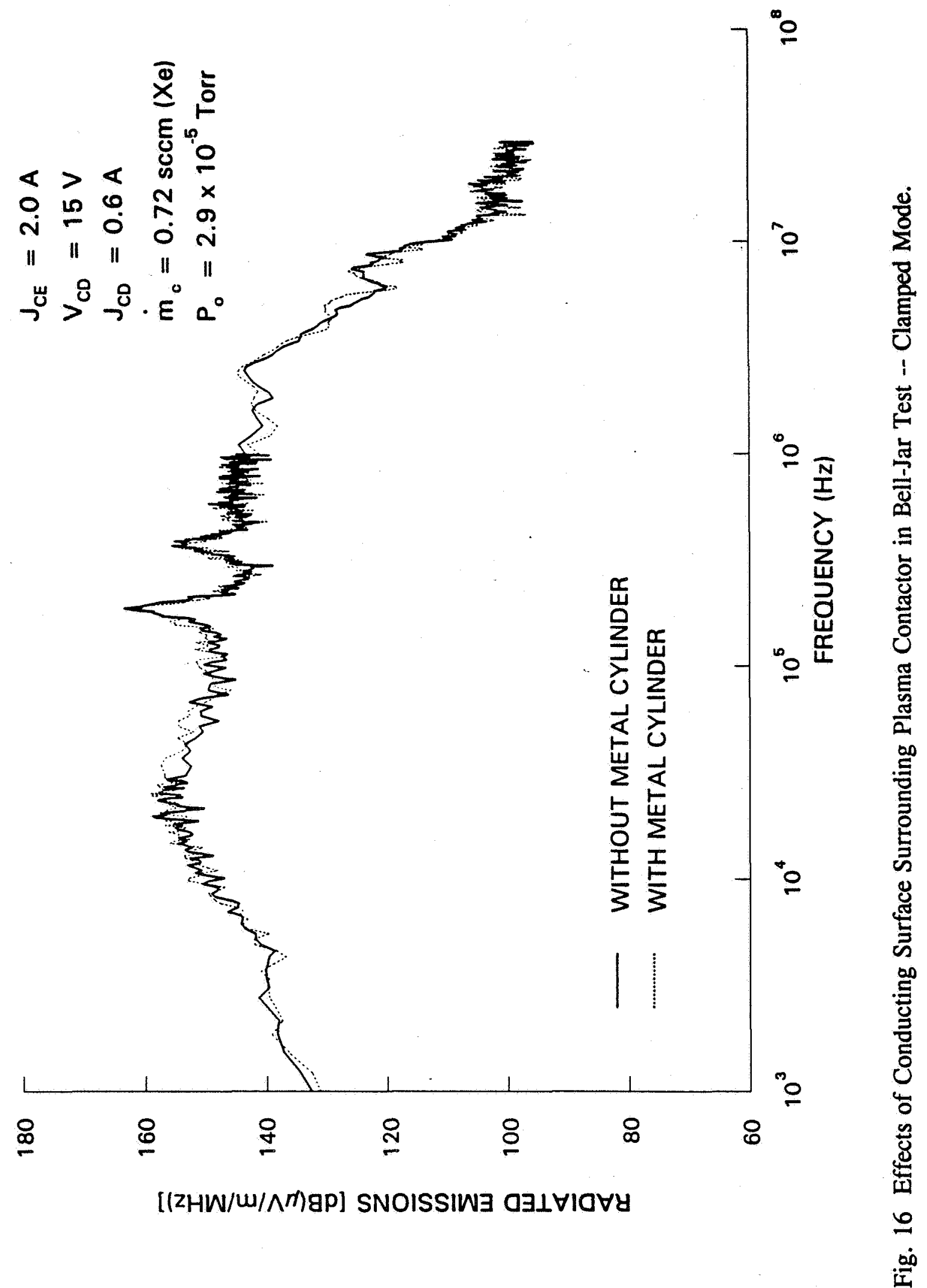


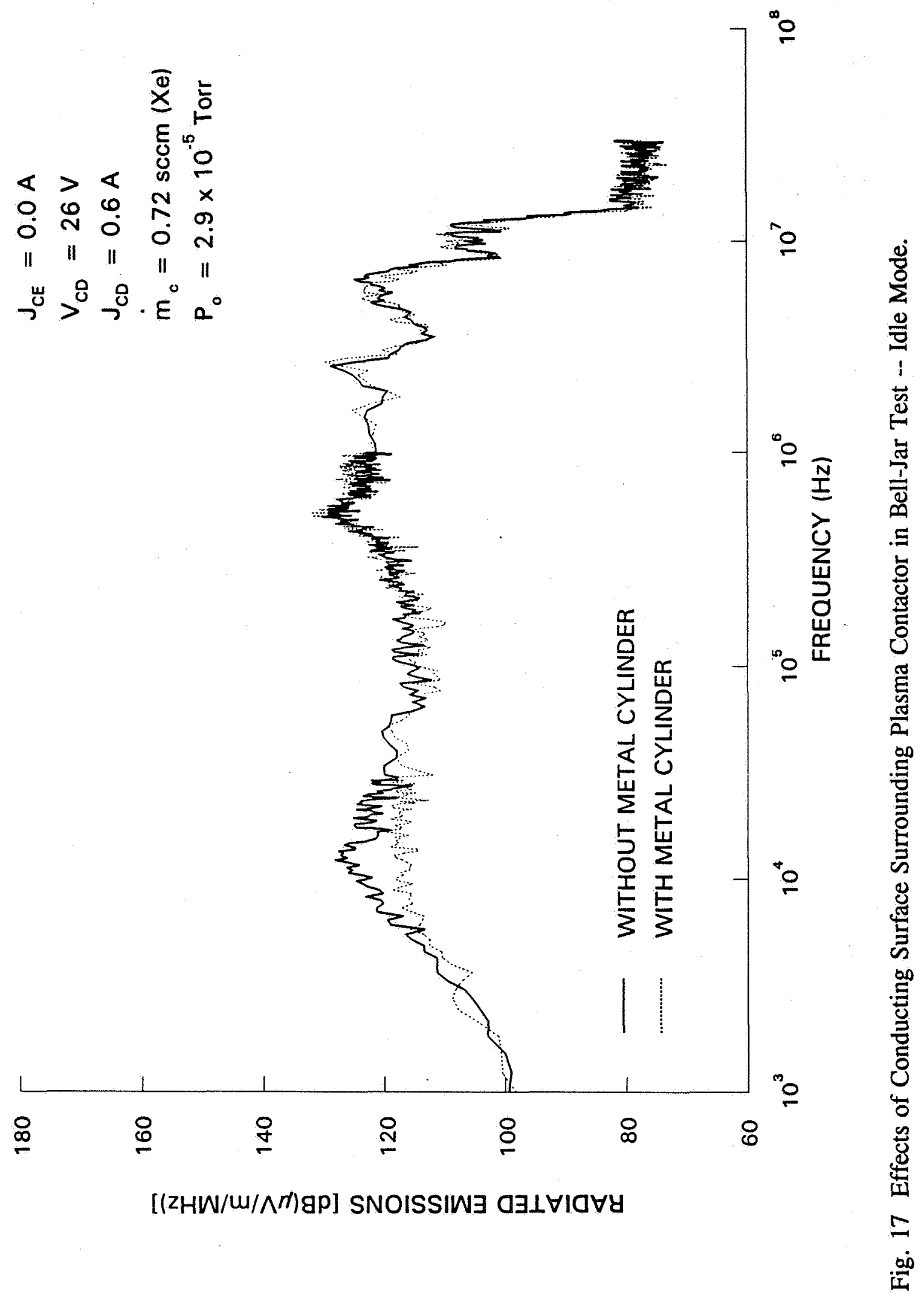


modest, reproducible difference in data near $10 \mathrm{kHz}$ the data are nearly identical. Since the reflective metal cylinder might induce EM wave reflections at some frequency, the results of Figs. 16 and 17 suggest that EM waves may not be the source of the noise sensed by the antenna at any of the frequencies investigated when a plasma is present, although this possibility is not excluded.

The results of EM-wave-reflection experiments show that caution must be exercised when interpreting RE data acquired in the vacuum-tank facility. Data collected at frequencies greater than $1 \mathrm{MHz}$ may not represent true contactor RE because of the interactive effects of EM waves with tank metallic surfaces. The results of the bell-jar experiment, however, indicate that no reflection effects are observed at any frequency when the contactor is operating in that facility. Extension of these results to the vacuum tank is difficult because of the geometrical differences between the two facilities, and also because of the different contactor operating conditions in each facility. It may be concluded, though, that EM wave interactions with the vacuum-tank metallic surfaces are probably not important except at the higher frequencies where the wavelengths are comparable to the tank dimensions.

\section{Noise Induced by Power Supplies}

Several experiments were performed to determine the extent to which RE and CE were induced by laboratory power supplies. Series-connected groups of lead-acid batteries were used to replace the conventional laboratory contactor-discharge, simulator-discharge, and bias power supplies. The laboratory equipment was set up so that the power supplies could be switched from conventional to battery power 
sufficiently quickly (a few seconds) so that significant changes in flow or discharge conditions were unlikely. Both RE and $\mathrm{CE}$ were measured using various combinations of battery and conventional power supplies to determine the baseline noise levels associated with the three power supplies. These tests were performed in the vacuum-tank facility with the altered configuration and the receiving antenna located $100 \mathrm{~cm}$ downstream of the contactor.

The effect of changing all of the aforementioned power supplies from conventional to battery on clamped-mode RE data is shown in Fig. 18 where it is seen that the data are essentially identical. Idle-mode RE data, shown in Fig. 19, are also very similar although there are minor differences near $500 \mathrm{kHz}$. Data were also acquired with all other mixes of conventional and battery power supplies and the results were the same, indicating that none of the conventional power supplies were contributing significantly to the noise sensed by the antenna. Conducted-emissions data were measured similarly and those results are seen in Fig. 20 as $d B(\mu \mathrm{A} / \mathrm{A}) \mathrm{v}$. frequency. At frequencies below $1 \mathrm{kHz}$ there are substantial differences between the two spectra, while at higher frequencies the differences are minor. By studying the effects of the various possible mixes of battery and conventional power supplies, it was discovered that the bias supply caused the higher CE noise levels shown below $1 \mathrm{kHz}$ in Fig. 20.

The results of these comparative experiments conducted with conventional and battery power supplies lead to the conclusion that plasma phenomena rather than power supplies are the sources of noise sensed by the antenna and that noise produced by the contactor is not amplified by the power supplies. The results also indicate 


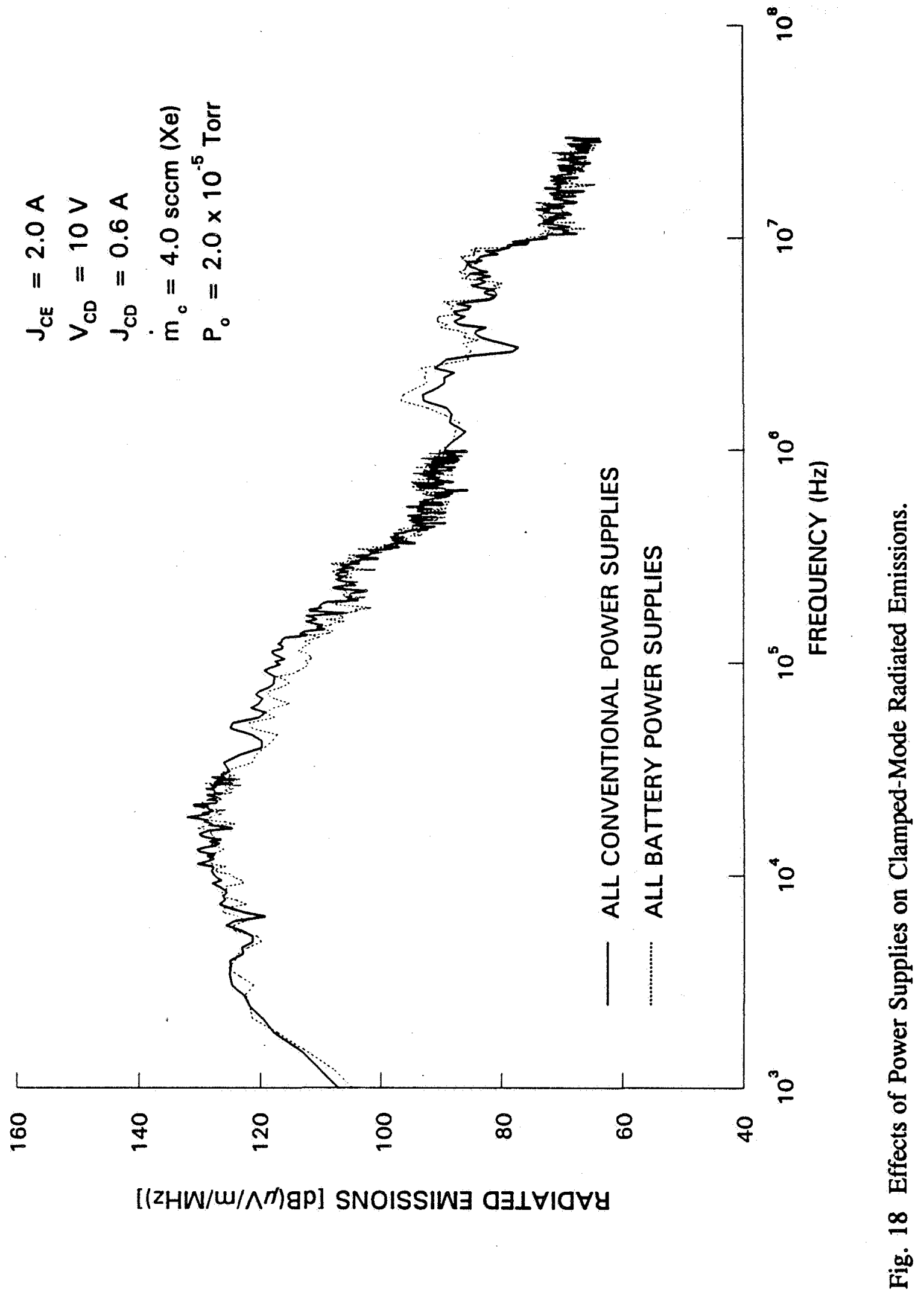




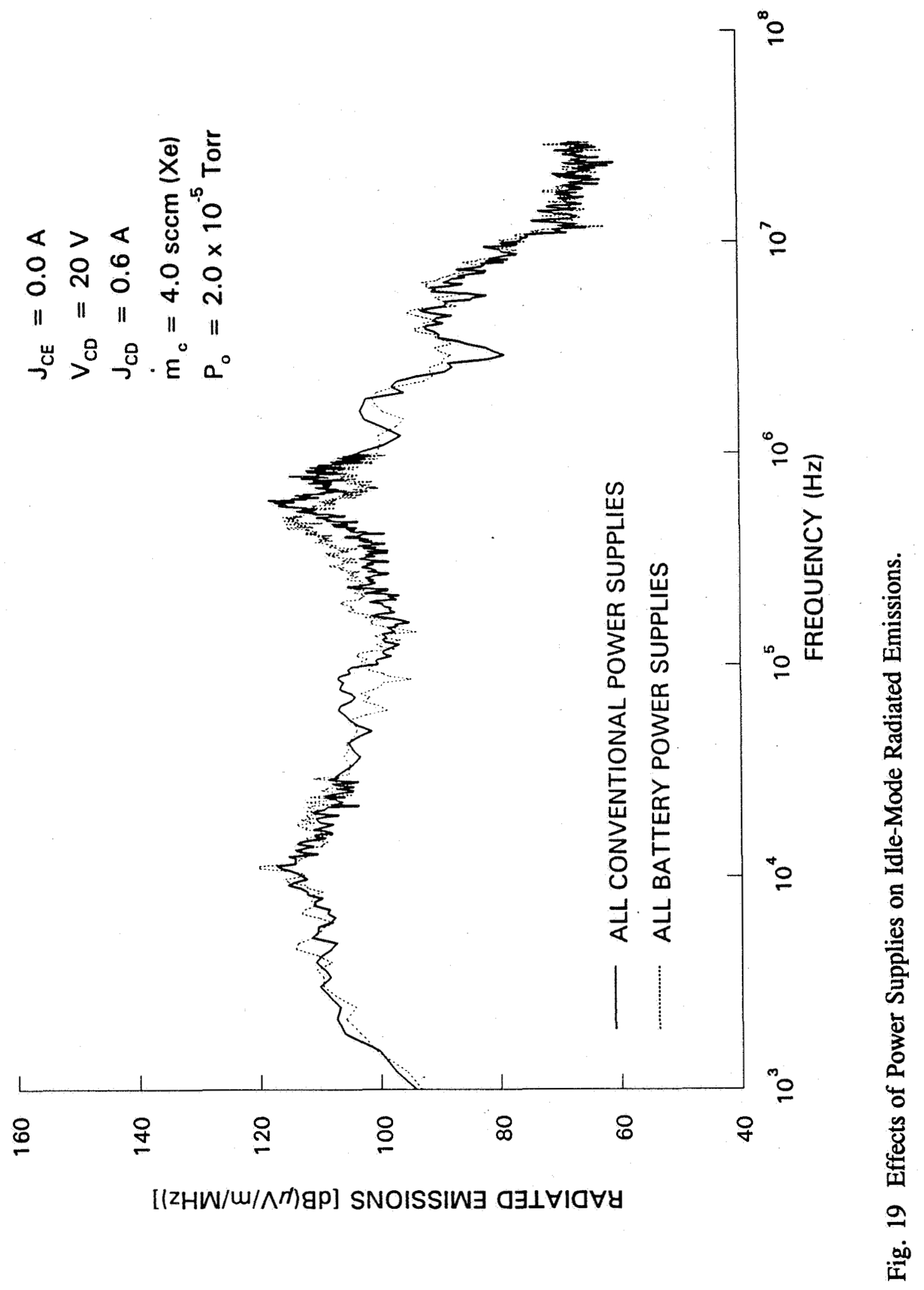




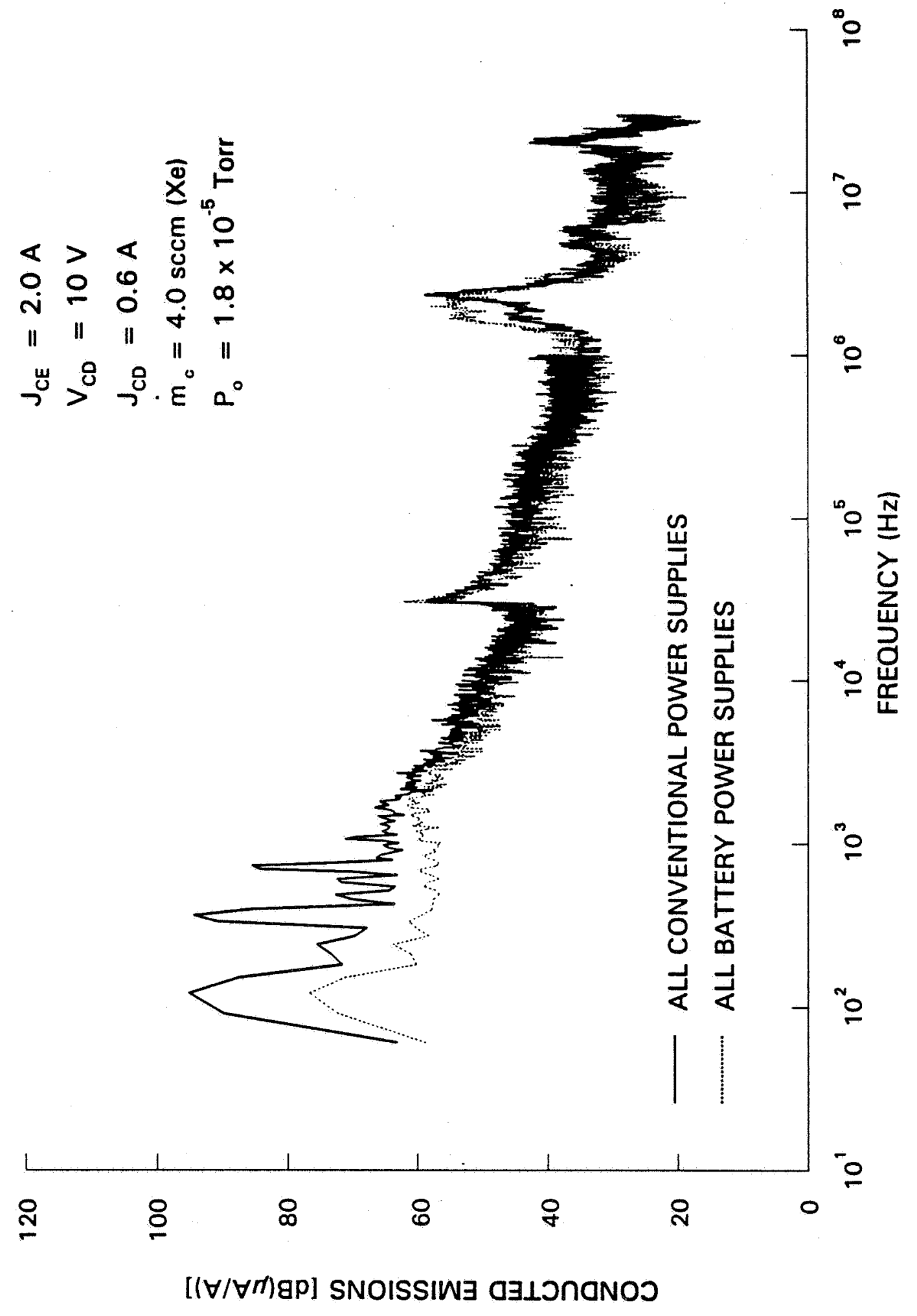

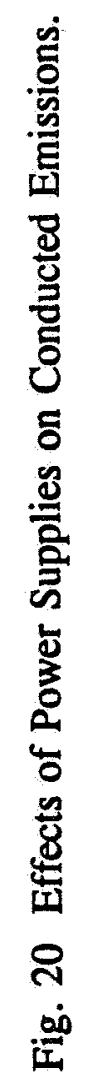


that, at frequencies between $1 \mathrm{kHz}$ and $30 \mathrm{MHz}$, phenomena related to the operation of the contactor and/or simulator are the source of CE.

\section{Relationship Between RE and CE}

Typical clamped-mode RE and CE data acquired with the contactor operating in the vacuum-tank facility are compared in the power spectral density format in Fig. 21. Here, CE data are presented as $\mathrm{mA}^{2} / \mathrm{Hz}$ and $\mathrm{RE}$ data as $(\mathrm{V} / \mathrm{m})^{2} / \mathrm{Hz}$. These two sets of data show the same general trends and are proportional in the frequency ranges 3 to $40 \mathrm{kHz}$, and 200 to $800 \mathrm{kHz}$. These trends suggest that there is a direct relationship between $\mathrm{RE}$ and $\mathrm{CE}$ at these low frequencies. At frequencies greater than $1 \mathrm{MHz}$, the correlation between the two sets of data appears to be poorer. Comparison of RE and $\mathrm{CE}$ data acquired when the contactor was emitting $1 \mathrm{~A}$ of current showed an even better correlation at frequencies less than $1 \mathrm{MHz}$.

A qualitative test in which the responses of the antenna and shunt resistor were compared in the time domain yielded the typical data shown in Fig. 22. These are simultaneously-acquired, raw RE (output of the antenna) and $\mathrm{CE}$ (voltage fluctuations across the shunt resistor) data from a bell-jar experiment. It can be seen that the general trends of both RE and CE data are very similar, and that several peaks occur in each set of data at the same times (e.g. near $22 \mu \mathrm{sec}$ and $34 \mu \mathrm{sec}$ ). The latter observation is important because it indicates the two signals are in phase at the lower frequencies. Although the correlation between $\mathrm{RE}$ and $\mathrm{CE}$ data measured in the vacuum tank was not as good as that for the data of Fig. 22, the signals still correlated reasonably well at lower frequencies. Encouraged by these results and by 


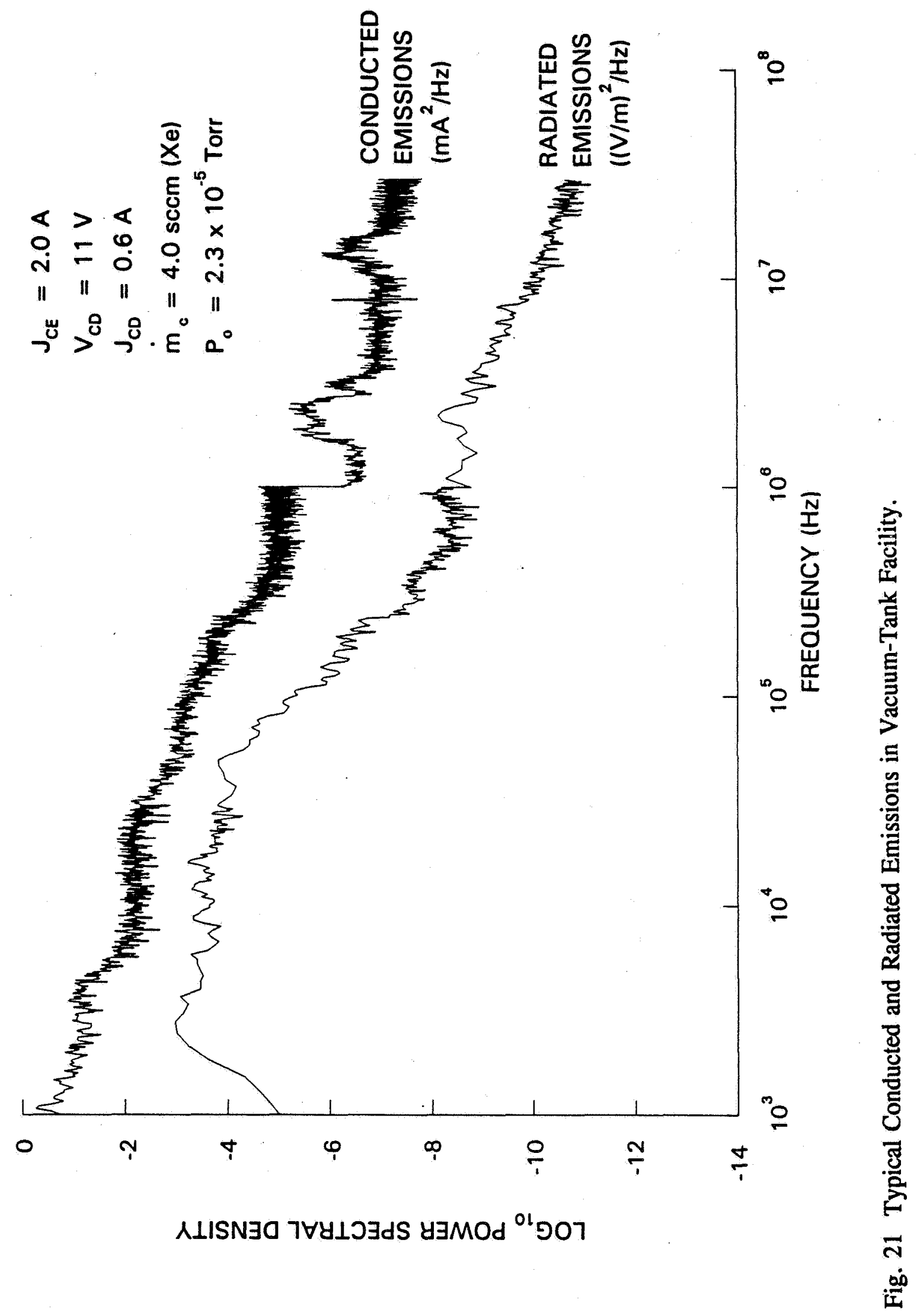


( $) ~ \exists a n \perp l 7 d W \forall \exists \supset$

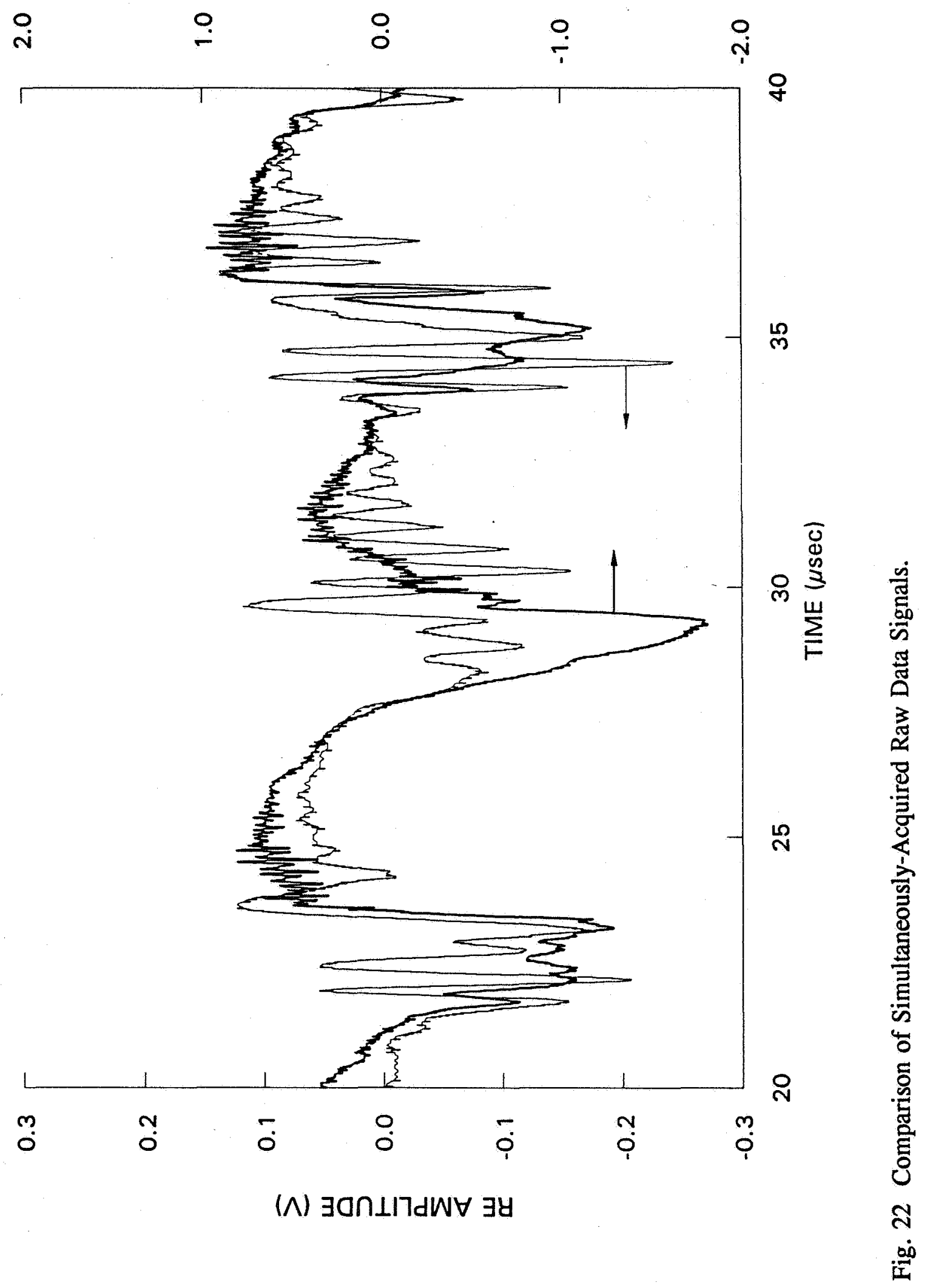


theoretical predictions, several experiments, described in the following sections, were undertaken to investigate the relationship between $R E$ and $C E$ at various frequencies.

\section{Electromagnetic Wave Broadcast Experiments}

Experiments were performed in the vacuum-tank facility to determine the nature of EM wave behavior in the contactor-plasma plume. The broadcast antenna, positioned in the tank as shown in Fig. 4, was used to transmit EM waves of a prescribed frequency into the contactor plasma and both RE and CE data were recorded. Representative RE data are shown in Fig. 23 for no-broadcast, 100-kHzbroadcast, and 10-MHz-broadcast cases. No signal can be seen above normal contactor noise (no-broadcast condition) at $100 \mathrm{kHz}$ for that broadcast condition. Large peaks can be seen, however, at the fundamental and the first harmonic for the $10 \mathrm{MHz}$ broadcast condition (the first harmonic is produced in the RF supply that powers the broadcast antenna and is unavoidable). Except for these peaks in the $10 \mathrm{MHz}$ broadcast test, the RE spectra for both broadcast tests are the same as for the no-broadcast condition. Additional tests were performed in which broadcast waves were successfully detected above the normal contactor noise at frequencies as low as $1 \mathrm{MHz}$. An understanding of the theory of the interaction between an electromagnetic wave and a plasma can help explain these results.

In a collisionless, uniform plasma there is a unique, critical frequency, called the plasma frequency, above which TEM waves will freely propagate through the plasma. At frequencies less than the plasma frequency, TEM waves will be completely reflected by the plasma. Recall that a collisionless plasma is a perfect 


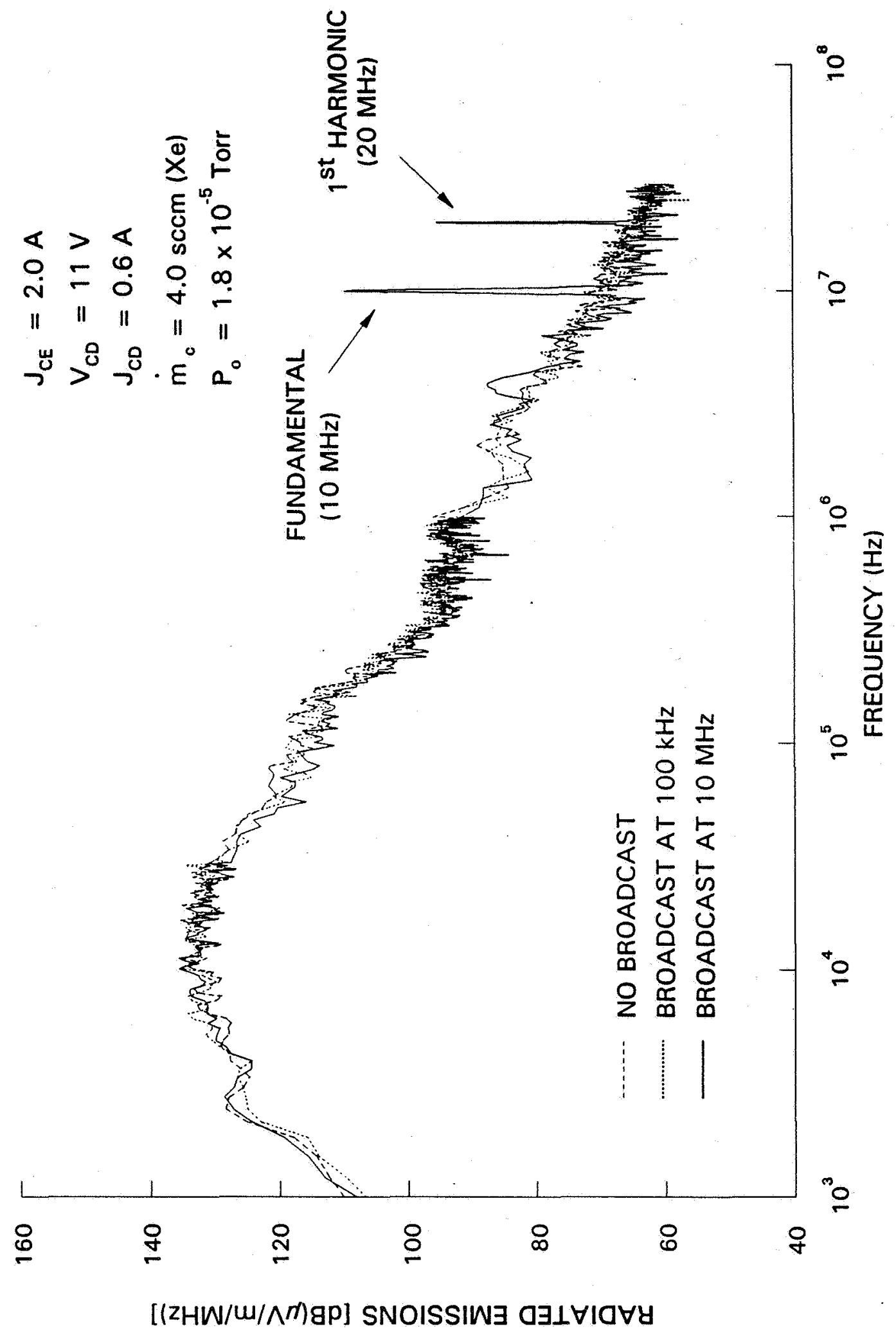

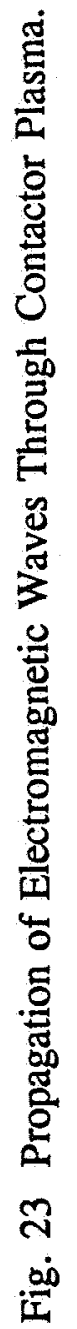


conductor and hence has zero skin depth [24]. If collisions are allowed, lowfrequency waves (compared to the plasma frequency) will not be completely reflected but will penetrate and be attenuated some distance into the plasma determined by the plasma skin depth. In a collisional, non-uniform plasma such as that produced by the contactor and simulator there will not be a sharp cutoff but rather a range of frequencies near the plasma frequency in which waves will be attenuated to varying degrees [8]. Certainly, very-low frequency waves will be entirely reflected and/or attenuated by the plasma and very-high frequency waves will pass completely through the plasma. The plasma frequency in the vacuum-tank varies with position in the tank, but is expected to be of the order of $10 \mathrm{MHz}$ on the tank centerline $1 \mathrm{~m}$ from the contactor [19]. Thus it is reasonable that the $10 \mathrm{MHz}$ signal is detected by the receiving antenna (and plausible that the $1 \mathrm{MHz}$ signal is, too, even though it will be attenuated by the plasma much more than is the $10 \mathrm{MHz}$ signal), while the $100 \mathrm{kHz}$ signal is not because it is reflected/attenuated completely by the plasma before reaching the receiving antenna. Recall, also, that these signals detected by the receiving antenna are near-field and will interact differently with the plasma than the TEM waves discussed here.

A closer analysis of the magnitude of the $100 \mathrm{kHz}$ signal, which was investigated at several receiving antenna locations in another experiment, supports this argument. During this experiment the broadcast antenna transmitted the $100 \mathrm{kHz}$ signal inside the tank when no plasma was present as well as when the contactor was operating in the clamped mode. In both cases the amplitude of the signal generator driving the broadcast antenna was the same. When the contactor was operating in the 
clamped mode, it was observed that a $100 \mathrm{kHz}$ signal above the normal contactor noise was not detected by the receiving antenna as its position was varied between 130 to $210 \mathrm{~cm}$ from the contactor. Since no $100 \mathrm{kHz}$ signal level was detected, the signal magnitude can be no greater than the magnitude of the plasma noise floor which masks it. The results of the two tests are shown in Fig. 24 where the mean plasma noise floor at $100 \mathrm{kHz}$ is plotted for the clamped-mode test and all data are normalized with respect to the maximum signal received in the no-plasma test. The data show that the clamped-mode plasma reduces the signal strength of the $100 \mathrm{kHz}$ signal being broadcast into it by at least two orders of magnitude at nearly all positions. This suggests that the signal is indeed reflected/attenuated by the plasma before it can reach the receiving antenna when the antenna is located between 130 and $210 \mathrm{~cm}$ from the contactor.

Conducted-emissions data were also investigated during EM wave broadcast tests. Figure 25 shows $\mathrm{RE}$ and $\mathrm{CE}$ data acquired when a $10 \mathrm{MHz}$ signal was being broadcast in the vacuum tank and the contactor was operating in the clamped mode. The $10 \mathrm{MHz}$ broadcast fundamental and the first harmonic are apparent in both spectra, and the second harmonic is visible in the CE spectrum. The two CE peaks near $100 \mathrm{kHz}$ are due to signal aliasing, a common numerical byproduct of FFT analysis $[19,25]$, and thus are not physically present in the contactor-current-return line. Tests at other frequencies showed that broadcast EM waves of frequencies of $1 \mathrm{MHz}$ or greater appeared as current fluctuations in the contactor-current-return line while those of lesser frequency did not. This important result indicates that a $1-\mathrm{MHz}$ or greater frequency electrical disturbance in the plasma, e.g. a broadcast or naturally 


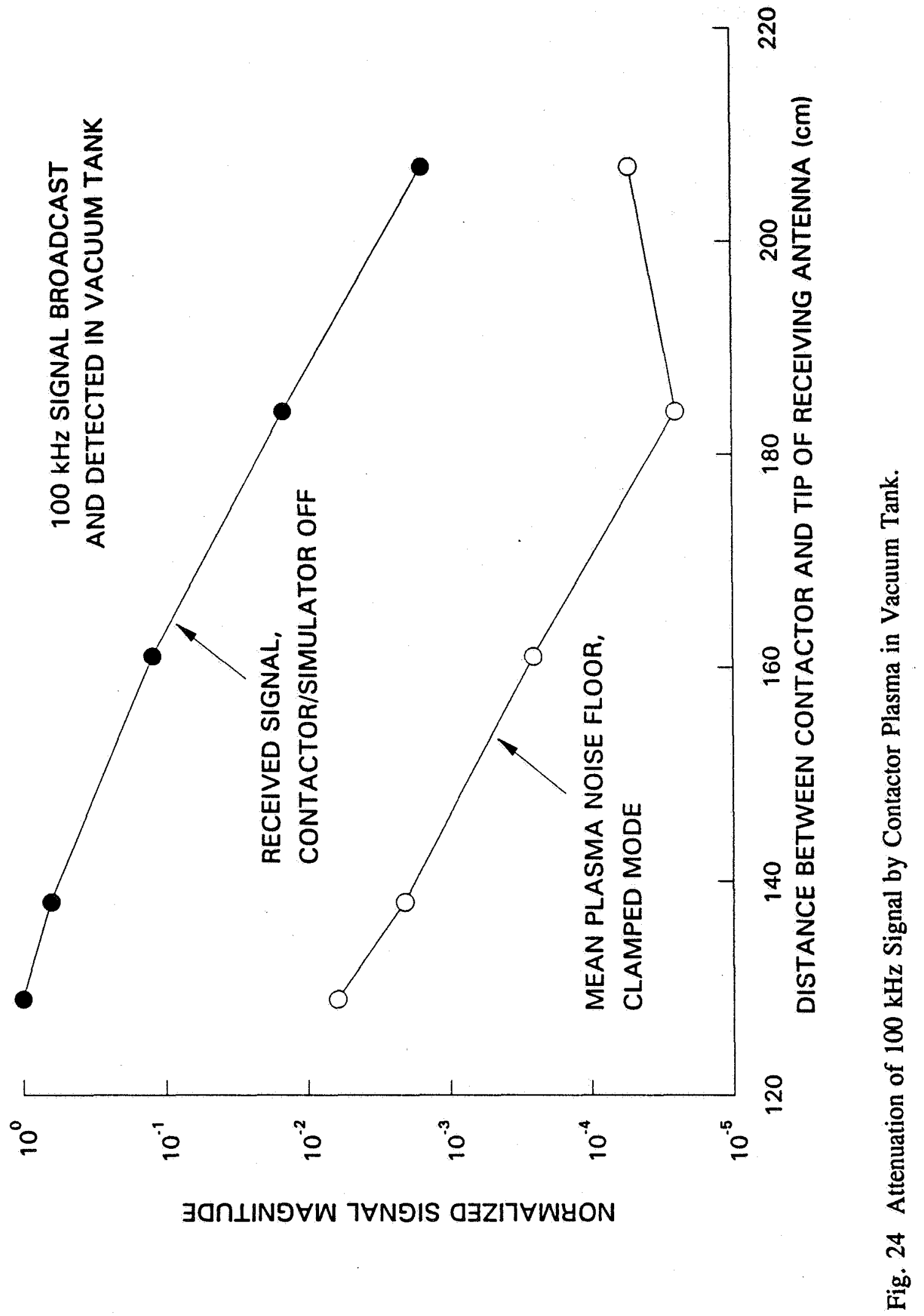




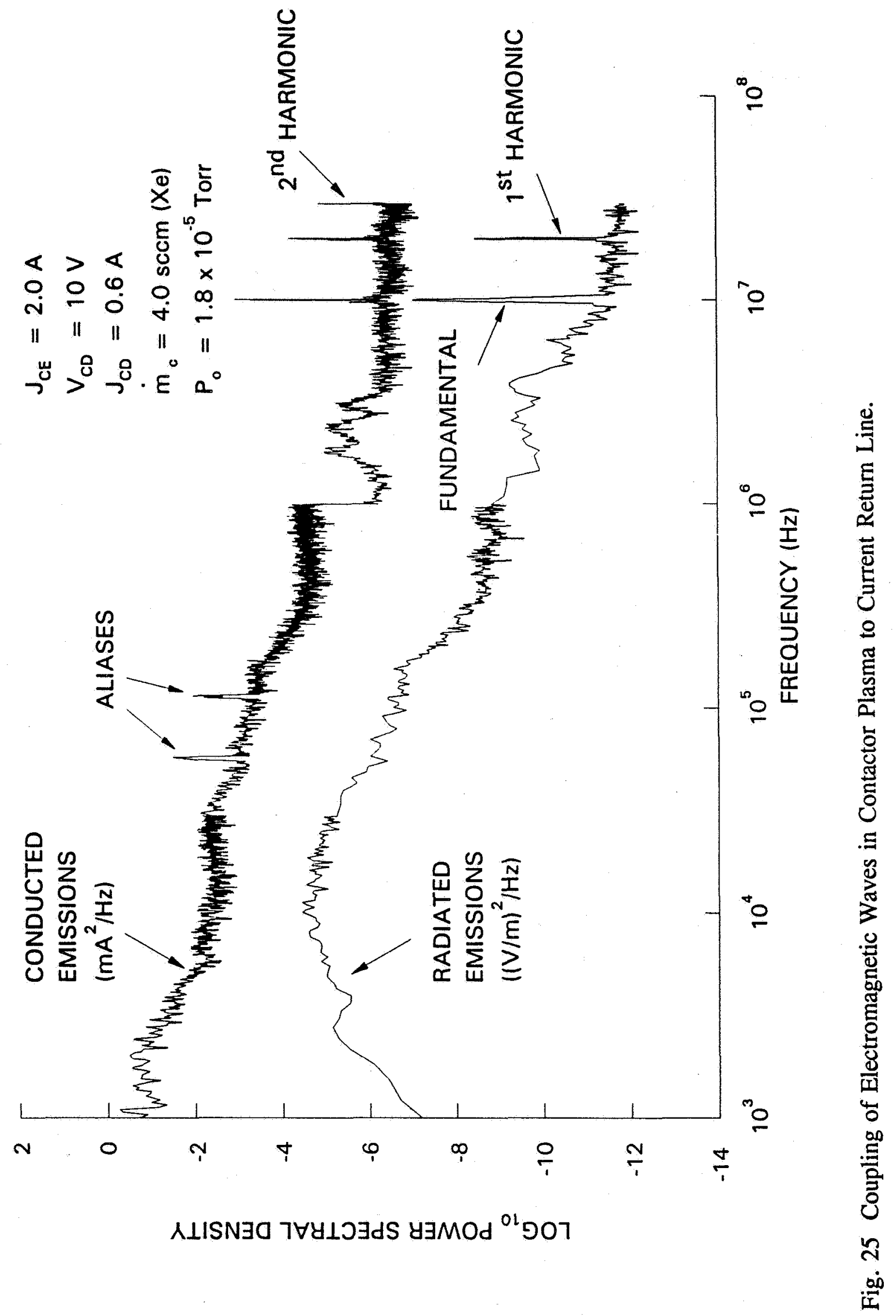


occurring EM wave, may couple through the simulator/tank walls and be transmitted to the hard-wired electrical circuit.

The results of the EM wave broadcast experiments indicate that EM waves of frequency $1 \mathrm{MHz}$ and greater are able to pass through the contactor plasma. Thus, the noise sensed by the antenna at frequencies above $1 \mathrm{MHz}$ may be partially or entirely remotely-produced noise. At lower frequencies, EM waves are not able to pass through the plasma. Thus, the noise sensed by the antenna at these frequencies is probably not produced at locations far from the antenna. It must, therefore, be produced at locations relatively near the antenna.

\section{Induced Current Fluctuation Experiments}

A vacuum-tank experiment was conducted where, instead of broadcasting EM waves into the plasma, current fluctuations were superimposed in the contactorcurrent-return line using the air-core transformer depicted in Fig. 7. Noise data were recorded as a current fluctuation was induced in the return line at a prescribed frequency. Figure 26 shows RE and CE data acquired when a $100 \mathrm{kHz}$ current fluctuation was superimposed in the return line. In the CE spectrum, the fundamental is obvious and some higher harmonics and noise generated by the RF source are also observed. The RE spectrum shows a single peak at the fundamental frequency. The test was repeated for superimposed current fluctuations of frequencies as high as $10 \mathrm{MHz}$ and as low as $50 \mathrm{kHz}$. In each case, correlated peaks were observed in RE and CE spectra at the fundamental frequency. 


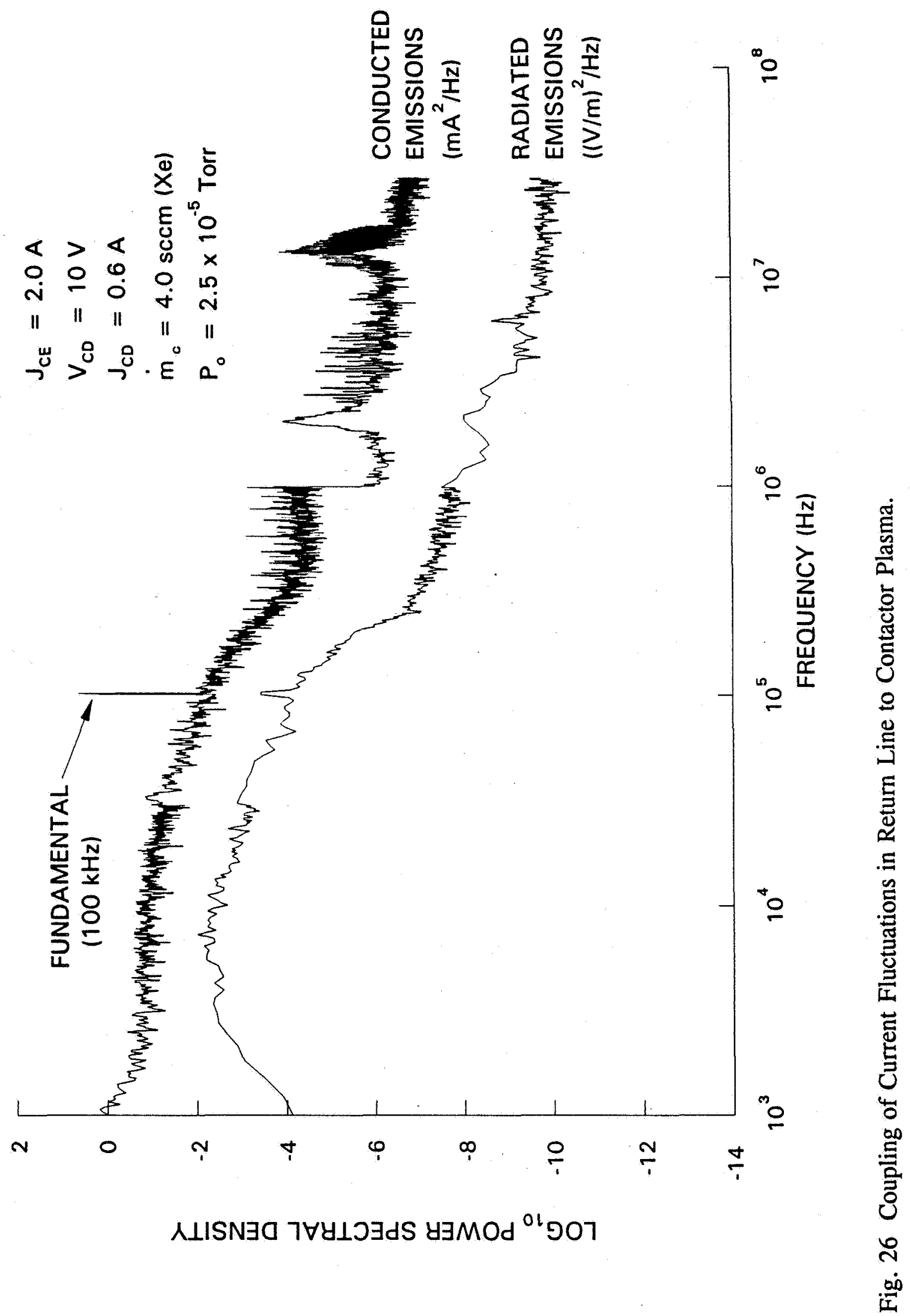


The results of this experiment show that current fluctuations in the contactorcurrent-return line are communicated through the contactor plasma to the receiving antenna where they can be detected as electric-field fluctuations in the plasma. An important result can be obtained by considering these results in the light of the results obtained when EM waves were broadcast into the plasma. Electromagnetic waves broadcast at frequencies less than $1 \mathrm{MHz}$ were unable to pass through the plasma to the receiving antenna, yet current fluctuations at those frequencies induced strong receiving-antenna signals. If the superimposed low-frequency current fluctuations had been completely transformed into EM waves at the contactor, they would not be expected to reach the receiving antenna, based on the results of the broadcast tests. If they remained current fluctuations, however, and traveled as currents through the resistive medium (i.e. the plasma) to the vicinity of the antenna, they could be detected by the antenna. Thus it appears, as theoretical models predict, that lowfrequency noise detected by the antenna is locally produced, rather than remotely produced.

\section{Plasma Exclusion Region Experiments}

Another vacuum-tank experiment was performed in which the ambient plasma was removed from the immediate vicinity of the receiving antenna monopole. This was accomplished by enclosing the monopole in a 130 -mm-diameter plexiglas cylinder, creating a $65-\mathrm{mm}$-radius region of plasma exclusion between the monopole and the outer surface of the cylinder. The cylinder was closed at both ends except for a small hole on the centerline at one end which allowed insertion of the monopole. 
Results of this experiment were compared to those where the antenna was insulated from the plasma by wrapping it with a thin dielectric material which provided a $0.2-\mathrm{mm}$ radial plasma exclusion region.

Clamped-mode noise sensed by the antenna with the 65 -mm-radius exclusion region are compared to those sensed with the 0.2 -mm-radius exclusion region in Fig. 27. At frequencies greater than about $200 \mathrm{kHz}$, RE levels with the larger exclusion region are about an order of magnitude below those with the smaller exclusion region. At frequencies less than $200 \mathrm{kHz}$, however, a reduction in RE levels of up to three orders of magnitude is obtained with the $65-\mathrm{mm}$ exclusion region. The plexiglas cylinder surrounding the monopole does not significantly attenuate any incident EM waves which are created far from the antenna, thus the difference between RE levels observed with the two separate plasma exclusion regions must be related to a local phenomenon. Specifically, it is argued that current fluctuations in the plasma are closer to the antenna with the smaller exclusion region and that this causes RE to be greater than those sensed with the larger exclusion region where the fluctuations are further from the antenna. This behavior is consistent with the idea that the noise measured by the antenna at lower frequencies (less than $1 \mathrm{MHz}$ ) is locally produced, not remotely produced. Note that it is also apparent that $\mathrm{RE}$ at frequencies greater than $1 \mathrm{MHz}$ are partially due to locallyproduced noise.

These observations were further supported when a comparison of RE data acquired with the 0.2 and $65-\mathrm{mm}$ exclusion regions was made with the contactor operating in the idle mode. These results are shown in Fig. 28 where, at frequencies 


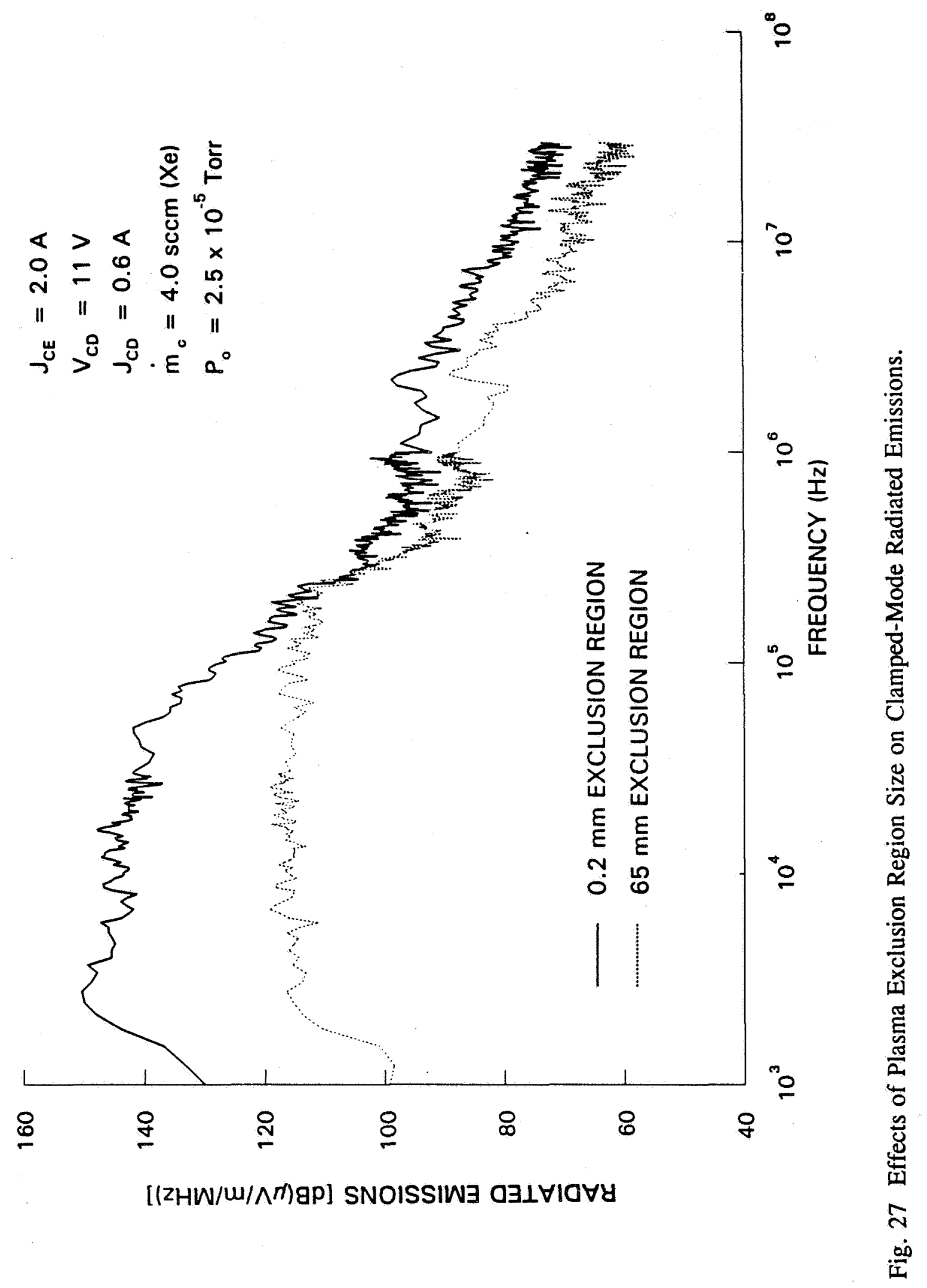




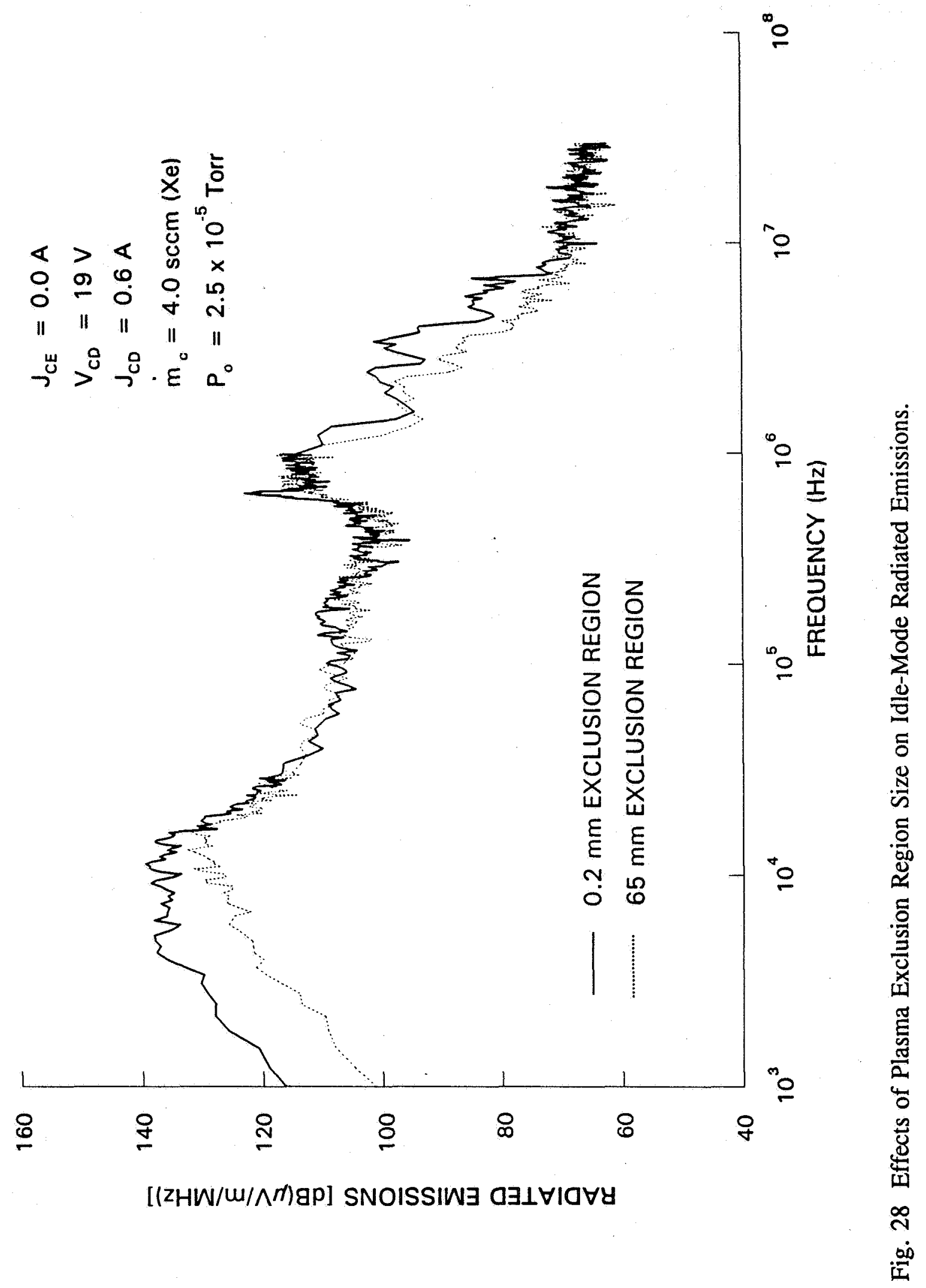


above $10 \mathrm{kHz}$, there are relatively minor differences between $\mathrm{RE}$ data for each exclusion region size. These results are again considered consistent with the theoretical model because current flow near the antenna is expected to be negligible in the idle mode and this would cause locally-produced RE to be much less significant in this case.

\section{Comparison of Bell-Jar and Vacuum-Tank Results}

The size of the plasma exclusion region surrounding the receiving antenna monopole can be increased further by performing experiments in the bell-jar facility, where the antenna is located outside of the vacuum boundary. Data were acquired in both the vacuum-tank and bell-jar facilities with the contactor operating under the standard conditions for each facility. In order to make the plasma environment as similar as possible between the bell-jar facility, which does not include a plasma source other than the plasma contactor, and the vacuum-tank, which has the simulator, all vacuum-tank data were acquired with the simulator turned off. The antenna monopole was isolated from the plasma by a thin layer of dielectric material for the vacuum-tank test, and in both facilities the tip of the antenna was pointed at and located $130 \mathrm{~cm}$ from the contactor along its centerline.

A comparison of RE data acquired when the contactor was operating in the idle mode was made first, and is shown in Fig. 29. The shapes of the curves are nearly identical and the bell-jar data are about two orders of magnitude less than the vacuum-tank data at frequencies up to about $1 \mathrm{MHz}$. At greater frequencies there are substantial differences in RE data, though the differences above $10 \mathrm{MHz}$ are probably 


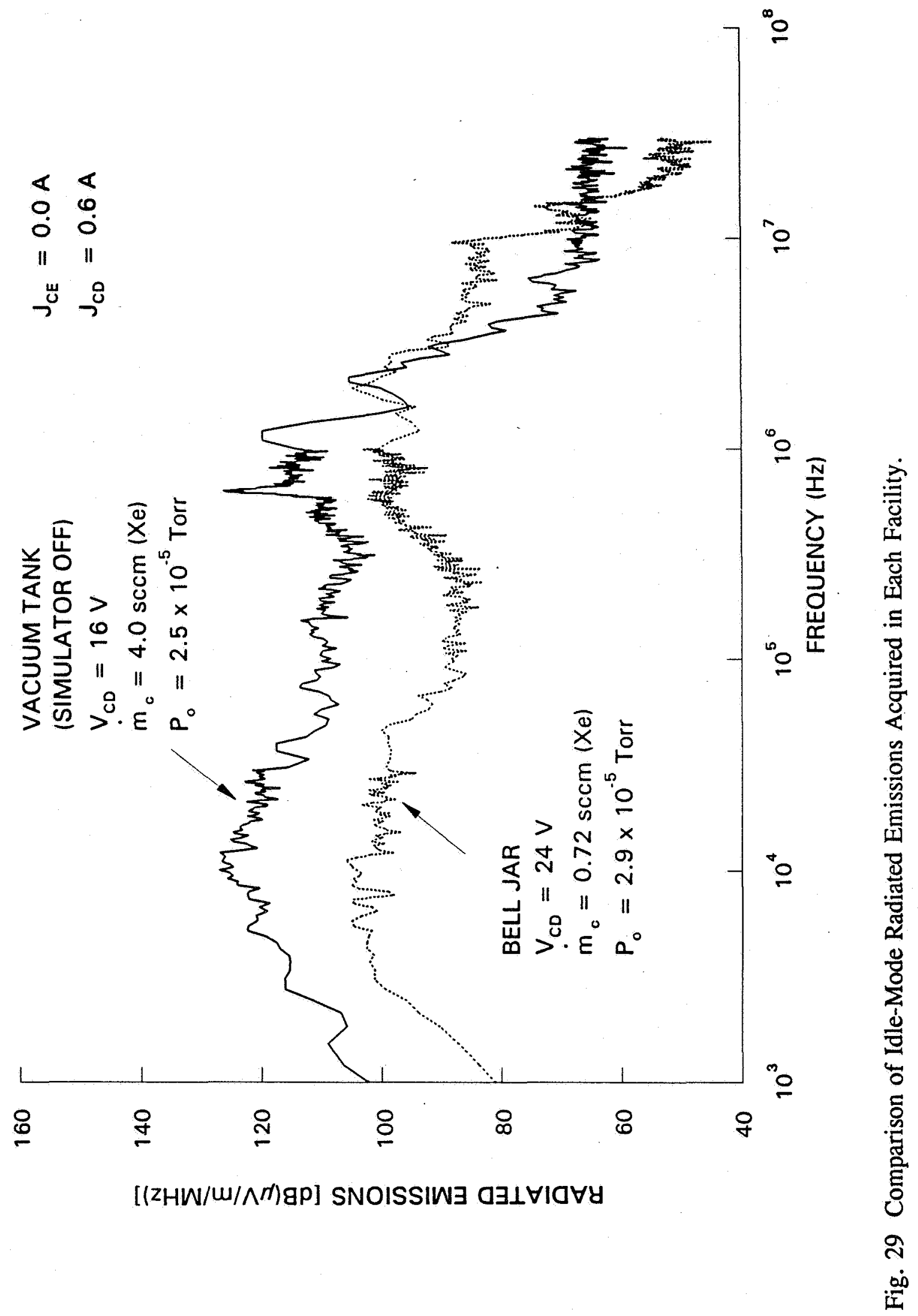


due to different noise floors for the two different antenna lengths used in the tests ( $1.8 \mathrm{~cm}$ for the bell jar and $0.5 \mathrm{~cm}$ for the tank). These similar shapes of the spectra below $1 \mathrm{MHz}$ suggest that similar physical processes are producing the noise observed below $1 \mathrm{MHz}$ when the contactor is emitting no current. For example, the discharge between the hollow cathode and the anode is most likely very similar in each vacuum chamber. The differences in magnitudes are probably related to geometrical and plasma environmental differences between test facilities. Recall the results of Fig. 28, where the shapes of the RE data are identical for different exclusion region sizes. This indicates that the differences in RE magnitude between test facilities seen in Fig. 29 is probably not due primarily to differences in the plasma-exclusion-region sizes associated with the facilities.

A comparison of RE data acquired when the contactor was operating in the clamped-mode in each facility is shown in Fig. 30. The RE data are markedly different in shape and magnitude at nearly all frequencies. Some of these differences are undoubtedly due to plasma environmental differences between the test facilities. These results are, however, not in conflict with the proposed model which indicates that the majority of lower-frequency RE noise is locally rather than remotely produced. In the bell-jar facility, electrons are collected on a small annular electrode and the current path may, therefore, be somewhat focused. In the vacuum-tank facility, on the other hand, diffuse current paths and electron flow adjacent to the antenna are expected as electrons are collected on the large area of the tank walls. Contactors emitting electrons along different current paths such as these could be expected to induce different electric-field environments. This would, in turn, give 


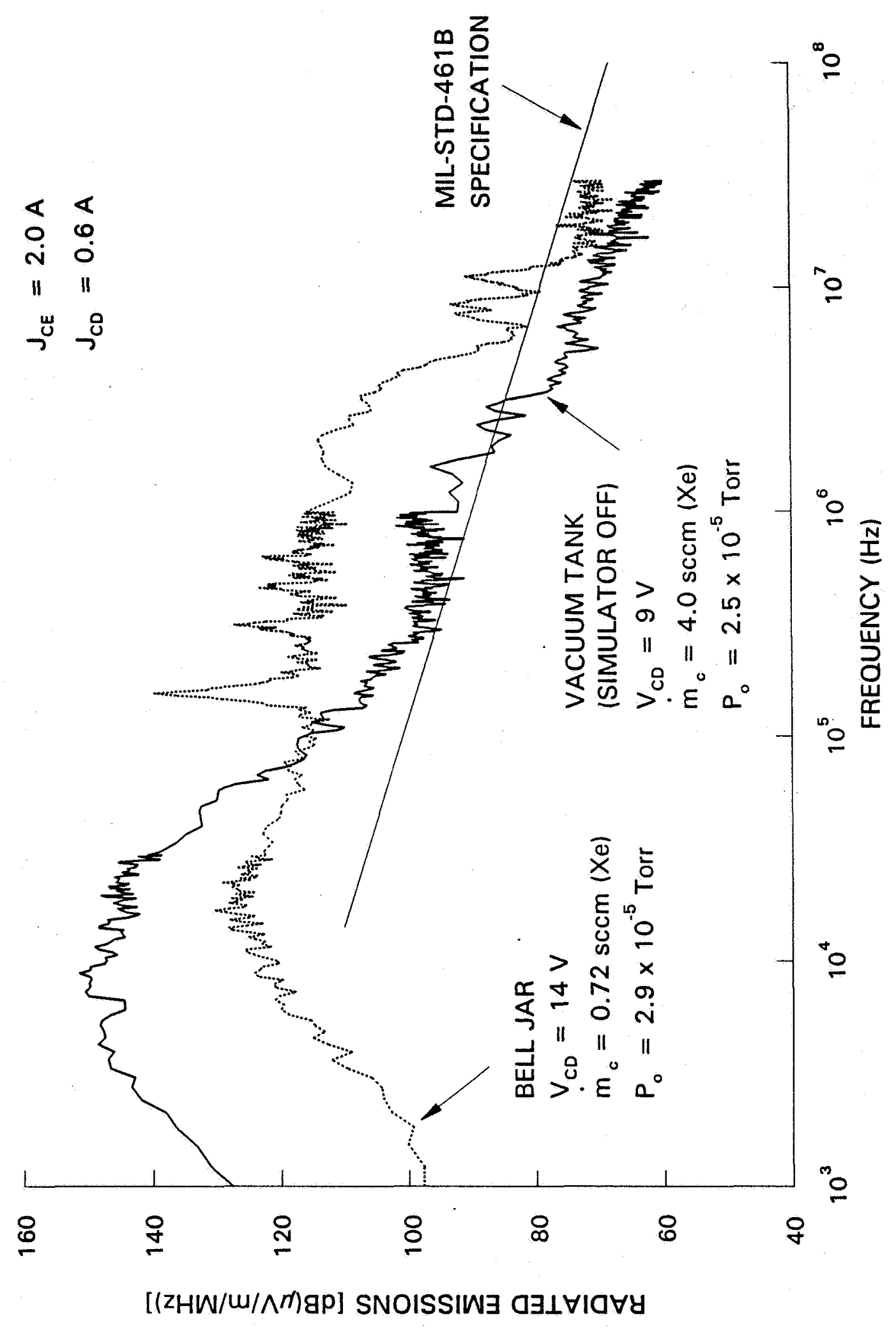

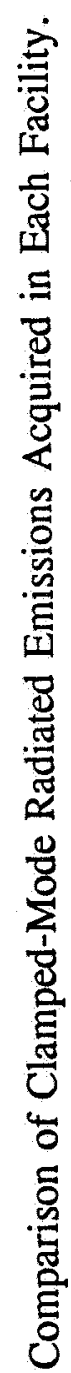

요

这 
rise to locally-produced contributions to the RE spectra that depend on the test facility and configuration. Recall that contactors emitting no electrons should have similar current flow patterns and should, therefore, show locally-produced RE that are similar regardless of the test facility being used. Indeed, Fig. 29 shows that the RE spectra are similar in shape at lower frequencies (less than $1 \mathrm{MHz}$ ). If the current patterns were known it should be possible to explain the differences between RE measured in each facility more precisely.

The RE data acquired in the vacuum-tank and bell-jar facilities are also compared to the military specifications [18] for RE in Fig. 30. Recall, the limit that is shown applies for broadband RE produced by equipment installed on spacecraft. Both sets of data in the figure appear to exceed the specifications at frequencies less than $3 \mathrm{MHz}$, but recall that at frequencies less than about $1 \mathrm{MHz}$ RE are locally produced, due to current fluctuations in the plasma, and that the military testing standards and specifications are not designed to cover this type of RE noise. The vacuum-tank and bell-jar data appear to meet the military specifications at frequencies greater than about $3 \mathrm{MHz}$ and $10 \mathrm{MHz}$, respectively, even though it has been shown that some of that noise is locally produced.

In order to obtain meaningful RE data, there must be methods for measuring $\mathrm{RE}$ that yield results independent of the measurement facility. The military standards for measuring RE [17] are deliberately designed in sufficient detail to produce RE spectra that are independent of test facility. An important observation drawn from the comparison of vacuum-tank and bell-jar RE data in this report is that the procedures for measuring RE of a contactor emitting electrons do not provide facility-independent 
results at any of the frequencies investigated. This is a consequence of the fact that fluctuating currents in the plasma near the antenna contribute substantially to the noise measured by the antenna, particularly at lower frequencies, and that such contributions to RE are not actually addressed by the military standards. 


\section{CONCLUSIONS AND RECOMMENDATIONS}

A monopole antenna which is used to measure RE while immersed in a plasma must be properly configured so that accurate measurements are obtained. It has been shown that it is necessary to prevent the monopole from coupling to the baseplate through the plasma and it was demonstrated that enclosing the monopole in a thin dielectric material is a sufficient and desirable method to accomplish this. Additionally, the monopole length must be sufficiently short so that saturation of the antenna amplifier circuitry does not occur. The monopole length must be determined for each experimental condition so that adequate antenna sensitivity is achieved while avoiding amplifier saturation.

Tests and analysis have indicated that EM wave interactions with the vacuumtank metallic surfaces can be significant at frequencies greater than $1 \mathrm{MHz}$. Thus, measured RE data may not represent true contactor RE at these higher frequencies. The results of bell-jar experiments, however, indicate that no reflection effects are observed over the complete frequency range investigated with the contactor operating in that facility. It was also determined that the conventional laboratory power supplies were not affecting either RE or CE at frequencies between $1 \mathrm{kHz}$ and $30 \mathrm{MHz}$.

Experimental results have shown a strong relationship between plasma contactor RE and CE. It has been demonstrated that electrical disturbances 
originating in the plasma can be communicated to the contactor-current-return line at frequencies greater than $1 \mathrm{MHz}$, and that current fluctuations in the return line at frequencies as low as $50 \mathrm{kHz}$ can be communicated to the plasma and be detected as electric-field fluctuations by an antenna. It has also been shown that at frequencies less than $1 \mathrm{MHz}$ the majority of RE sensed by the antenna are due to current fluctuations adjacent to the antenna monopole. Remote, radiative contributions to RE begin to become significant only at frequencies above $1 \mathrm{MHz}$.

Accurate measurements of contactor RE should be made in ground-based facilities which approximate, as closely as possible, the space environment. In space, a plasma contactor will emit current into the diffuse ambient space plasma through a large solid angle. This situation is approximated by electron collection on metallic tank walls that completely surround the contactor. It is believed that a large vacuumtank facility (not necessarily metallic) would be the best place to make measurements that can be used to infer contactor RE in space. In this type of facility the contactor plasma plume would be allowed to spread out into a large volume and current could be collected over a large area near the tank walls. The effects of EM wave reflection from tank metallic surfaces could be identified, if not quantified, using techniques similar to those employed in this study.

In order to extrapolate contactor performance measured in ground-based tests to space, $\mathrm{CE}$ should be measured at frequencies below about $1 \mathrm{MHz}$. These data should then be used in a model (such as that in ref. 20) that describes the space and spacecraft environments to compute RE that reflect the effects of both locally and remotely-produced noise. 


\section{FUTURE WORK}

The results of this investigation of plasma contactor noise production lead to several interesting possibilities for additional experiments. Although much evidence has been given to support the conclusion that the majority of noise measured by the antenna at frequencies less than $1 \mathrm{MHz}$ is due to current fluctuations adjacent to the antenna, more evidence could be obtained. For example, a direct measurement of the current fluctuations near the antenna with a small non-intrusive probe, and a correlation of those measurements with the noise sensed by the antenna, would likely provide even more conclusive evidence. Different means of collecting the electrons emitted by the plasma contactor could be used to force different paths of current flow, and the antenna measurements made in these new environments might be correlated to the current paths.

Additionally, more experiments could be performed with different shapes and sizes of plasma exclusion regions. These tests could provide insight to the physical proximity necessary for current fluctuations to have significant bearing on antenna measurements. Indeed, preliminary experiments of this type have been performed and it was observed that low-frequency noise reduction like that in Fig. 27 was achieved with exclusion regions much smaller than the $65-\mathrm{mm}$ one used to obtain those data. 
As an expansion upon the exclusion-region tests, and also in return to the motivating factor for measuring plasma contactor noise emissions, it may be possible to surround sensitive space-station electronic equipment near the plasma contactor with baffles which would divert current flow (and hence noise) from near the equipment. This simple step could eliminate a majority of the low-frequency noise which may interfere with the operation of those instruments. These types of experiments could easily be performed in a large vacuum-tank facility. 


\section{REFERENCES}

1. Williams, J. D., and Wilbur, P. J., "Experimental Study of Plasma Contactor Phenomena," Journal of Spacecraft and Rockets, V. 27, N. 6, Nov. 1990, pp. 634-641.

2. Williams, J. D. "An Experimental Investigation of Hollow Cathode-Based Plasma Contactors," NASA CR-187120, May 1991.

3. Katz, I., Jongeward, G. A., Gardner, B. M., Parks, D. E., Kuharski, R. A., and Mandell, M. J., "Preliminary Plasma Contactor Requirements," Progress Report, Dec. 17, 1992, Maxwell Laboratories Inc., S-Cubed Division, La Jolla, California.

4. Booth, Cristopher. J., ed., The New IEEE Standard Dictionary of Electrical and Electronics Terms, 5th ed., Institute of Electrical and Electronics Engineers, New York, 1993.

5. Motchenbacher, C. D., and J. A. Connelly, Low-Noise Electronic System Design, John Wiley and Sons, New York, 1993, Chaps. 1 and 12.

6. Paul, Clayton R., Introduction to Electromagnetic Compatibility, John Wiley and Sons, New York, 1992, Chap. 1.

7. Pettai, Raoul, Noise in Receiving Systems, John Wiley and Sons, New York, 1984, Chaps. 1-3, 11.

8. Spitzer, Lyman, Jr., Physics of Fully Ionized Gases, 2nd ed., Interscience Publishers, New York, 1962, Chap. 3.

9. Stix, Thomas H., The Theory of Plasma Waves, McGraw-Hill, New York, 1962, Chaps. 6, 7, and 9.

10. Fluke, John C., Controlling Conducted Emissions by Design, Van Nostrand Reinhold, New York, 1991, Chap. 1.

11. Mardiguian, Michel, Controlling Radiated Emissions by Design, Van Nostrand Reinhold, New York, 1992, Chap. 2. 
12. Balanis, Constantine A., Antenna Theory, Analysis and Design, John Wiley \& Sons, New York, 1982, Chaps. 2-4.

13. Department of Defense (US), "Definitions and System of Units, Electromagnetic Interference and Electromagnetic Compatibility Technology," MIL-STD-463, June 1977.

14. Jost, R. J., Neubert, T., Farley, D. T., Rodriguez, R., Calderon, C., Woodman, R., and Burch, J. L., "Radar Observations of ATLAS-1 Plasma Contactor Disturbances from Jicamarca Observatory," EOS, Geophysical Society Journal, V. 73, N. 43, Supplement, 1992, p. 412.

15. NASA Space Station Freedom Program Office, "Space Station Electromagnetic Techniques," SSP 30238, Reston, Virginia, March 15, 1991.

16. NASA Space Station Freedom Program Office, "Space Station Electromagnetic Emission and Susceptibility Requirements for Electromagnetic Compatibility," SSP 30238, Reston, Virginia, March 15, 1991.

17. Department of Defense (US), "Electromagnetic Interference Characteristics, Measurement of," MIL-STD-462, July 1967.

18. Department of Defense (US), "Electromagnetic Emission and Susceptibility Requirements for the Control of Electromagnetic Interference," MIL-STD-461B, April 1980.

19. Buchholtz, B. W., "An Investigation of Conducted and Radiated Emissions from a Hollow-Cathode Plasma Contactor," NASA CR-191172, July 1993.

20. Jongeward, G., Mandell, M. J., Katz, I., Buchholtz, B. W., Snyder, J. S., and Wilbur, P. J., "Conductive Nature of Low Frequency ( $<<1 \mathrm{MHz}$ ) Electromagnetic Fields Generated by a Hollow Cathode Plasma Contactor," AIAA Paper 94-3313, June 1994.

21. Katz, I., Barfield, J. N., Burch, J. L., Marshall, J. A., Gibson, W. C., Neubert, T., Roberts, W. T., Taylor, W. W. L., and Beattie, J. R., "Interactions Between the Space Experiments with Particle Accelerators Plasma Contactor and the Ionosphere," Journal of Spacecraft and Rockets, V. 31 , N. 6 , Nov 1994, pp. 1079-1084.

22. Williams, J. D., and Wilbur, P. J., "Plasma Contactor Research - 1989," NASA CR-185212, Feb. 1990, pp. 39-50.

23. Rizzi, Peter A., Microwave Engineering, Prentice Hall, Englewood Cliffs, New Jersey, 1988, Chap. 9. 
24. Narayana Rao, Nannapaneni, Elements of Engineering Electromagnetics, 3rd ed., Prentice Hall, Englewood Cliffs, New Jersey, 1991, Chap. 6.

25. Bendat, Julius S., and Allan G. Piersol, Random Data, Analysis and Measurement Procedures, 2nd ed., John Wiley and Sons, New York, 1986, Section 10.3.2. 


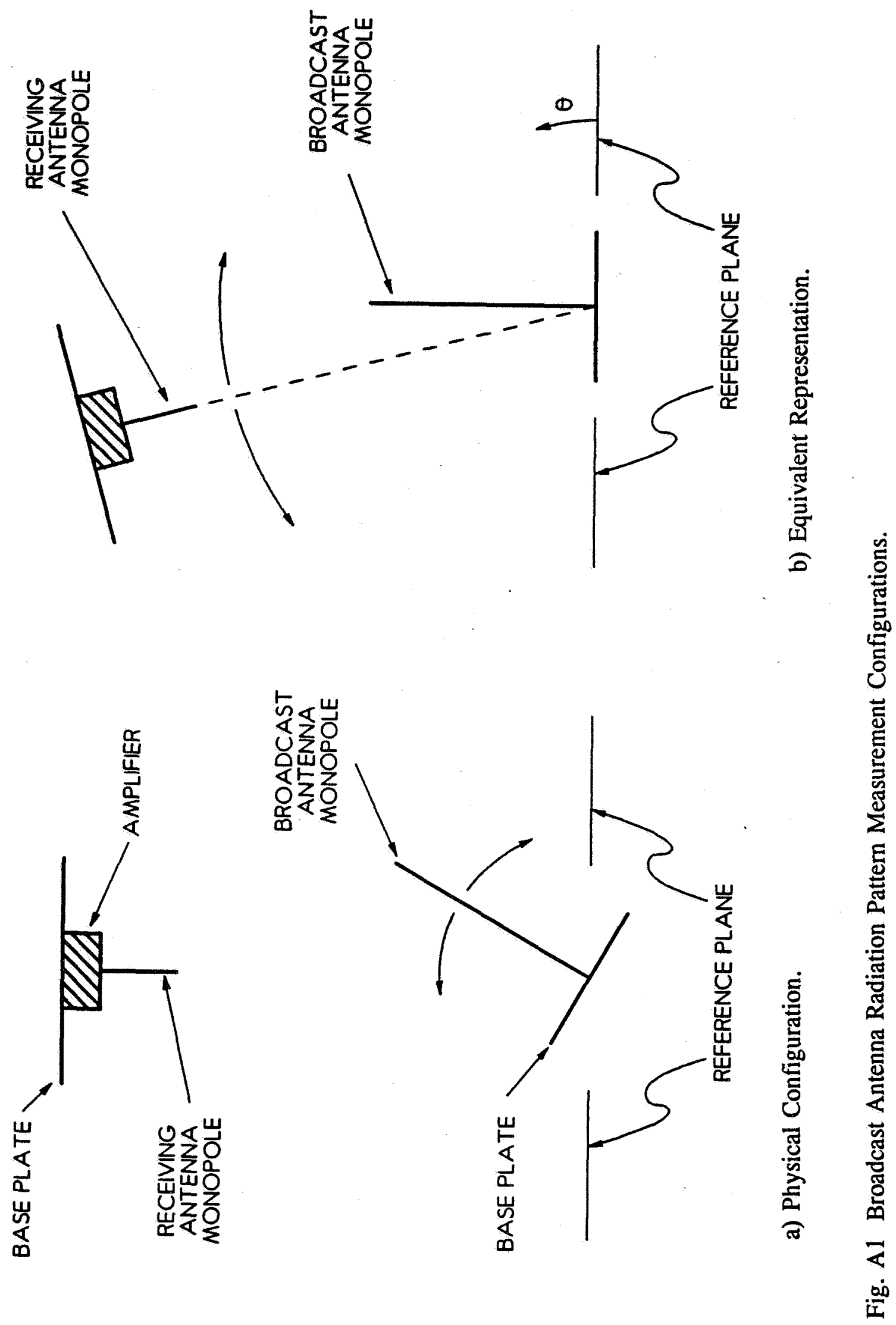




\section{APPENDIX A \\ Measurement of Broadcast Antenna Radiation Pattern}

The broadcast antenna used in the experiments described in this report was built in the CSU laboratory specifically for these experiments. The antenna calibration factor (used to determine broadcast signal strength from input signal strength) was unknown, but it was not needed for the experiments that were

performed. Several tests were conducted, however, to determine the radiation pattern of the broadcast antenna at different frequencies. The antenna radiation pattern is a two-dimensional, graphical representation of the radiated electric-field-fluctuation amplitudes in the space surrounding the antenna [12]. These tests were conducted to determine if the radiation pattern might be frequency dependent and might as a result complicate the interpretation of the experimental results.

The pattern-measurement experiment was conducted in a large indoor bay adjacent to the laboratory where reflections from surrounding objects could be minimized. In this area the surfaces closest to the broadcast and receiving antennae capable of reflecting EM waves were more than four meters away. The base plates of the antennae were $133 \mathrm{~cm}$ apart and the antennae were configured as shown in Fig. Ala. Note that under these conditions the receiving antenna will be measuring the near-field radiation patterns of the broadcast antenna. The tests were conducted 
by fixing the receiving antenna and rotating the broadcast antenna through $180^{\circ}$ about the intersection of the antenna monopole with the base plate. When the base plate of the broadcast antenna was in the reference plane the antennae monopoles were colinear. A signal of a given frequency was transmitted by the broadcast antenna, the amplitude of the signal sensed by the receiving antenna was recorded, and the broadcast antenna was then turned to a new angle and the process was repeated. This experimental procedure was followed because it is less complicated than the equivalent procedure of rotating the receiving antenna about the broadcast antenna, shown in Fig. Alb. The results of radiation pattern measurement are generally described graphically from the perspective of the latter configuration.

Typical results of the experiment are shown in Fig. A2a for three broadcast conditions. In this figure dimensionless signal amplitude is plotted on the radial axis and the polar angle $\theta$ as defined in Fig. Alb is measured from the reference plane to the dotted line shown. It is evident from the data in Fig. A2a that the broadcast antenna radiation patterns are nearly identical for frequencies of $1 \mathrm{MHz}, 100 \mathrm{kHz}$, and $10 \mathrm{kHz}$. Note that the patterns are symmetric about the line $\theta=90^{\circ}$ even though the indoor laboratory bay is a highly asymmetric environment and the broadcast antenna points at different surrounding objects in the bay as it is rotated (Fig. Ala). This clearly indicates that reflection of EM waves from the surroundings was not a factor in this experiment. Radiation patterns for broadcasts of 5, 10 and $25 \mathrm{MHz}$ are shown in Fig. A2b. Again the patterns are symmetrical, but they depart from the common patterns of Fig. A2a. As $\theta$ is varied from $90^{\circ}$ to $0^{\circ}$ the signal amplitude does not decrease as it did for the signals of Fig. A2a. Instead, the amplitude of the 


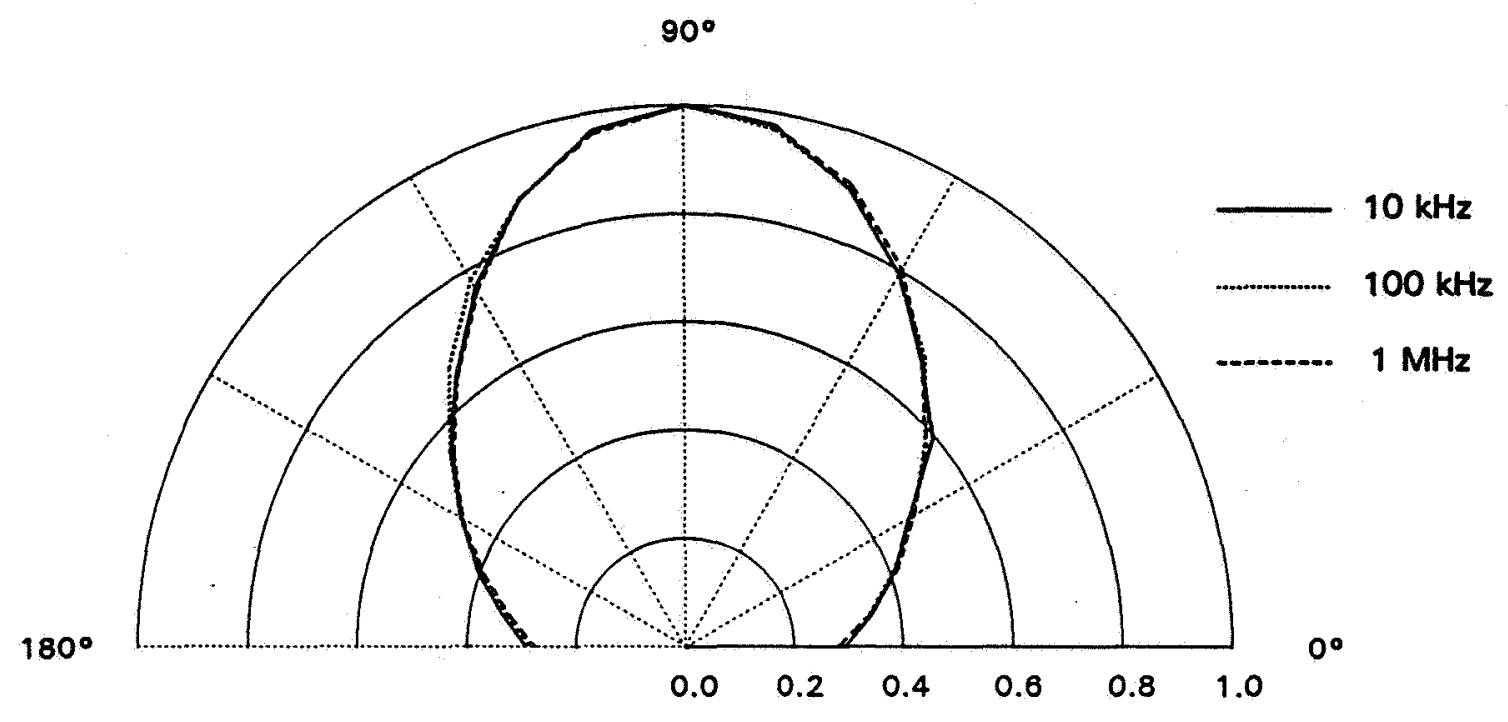

a) Radiation Patterns for Lower Frequencies.

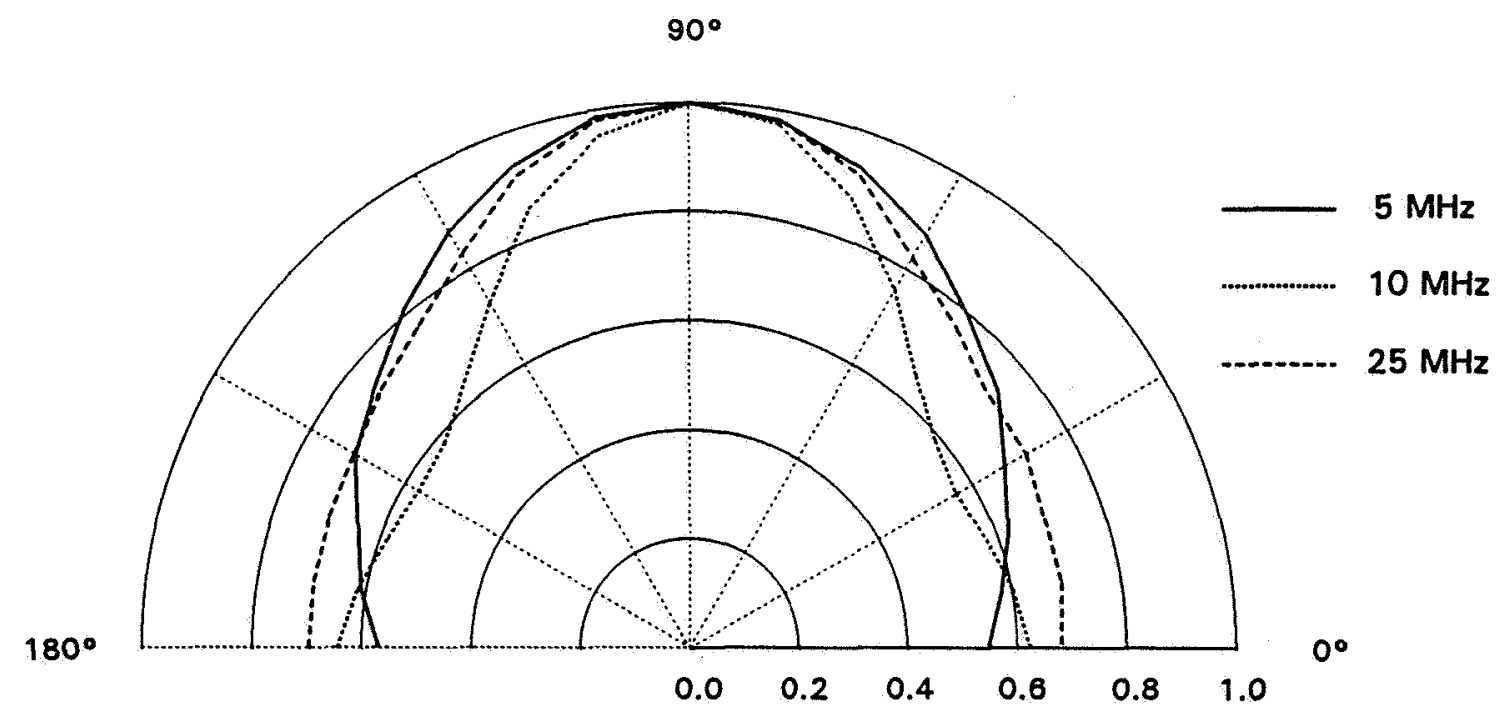

b) Radiation Patterns for Higher Frequencies.

Fig. A2 Broadcast Antenna Radiation Patterns. 
$10 \mathrm{MHz}$ signal decreases steadily and then increases, and the $25 \mathrm{MHz}$ signal decreases until $\theta \approx 45^{\circ}$ and then remains fairly constant. The amplitude of the $5 \mathrm{MHz}$ signal is much larger than those in Fig. A2a, but follows the same trends.

Note that at each of the frequencies investigated, there are no radiation 'lobes' which are portions of the radiation pattern bounded by relatively weak radiation intensity [12]. The presence of a lobe in the broadcast antenna radiation pattern could, if no EM wave reflections occurred inside of the vacuum tank, produce results from no-plasma broadcast tests that indicated significant reflection effects. 
Public reporting burden for this collection of intormation is estimated to average 1 hour per response, including the tirne for reviewing instructions, searching existing data sources. galhering and Davis Highway. Suite 1204, Arlington, VA 22202-4302, and to the Office of Management and Budget, Paperwork Reduction Project (0704-0188), Washington, DC 20503.

\begin{tabular}{|l|l|l|}
\hline 1. AGENCY USE ONLY (Leave blank) & $\begin{array}{c}\text { 2. AEPOAT DATE } \\
\text { March } 1996\end{array}$ & $\begin{array}{r}\text { 3. REPOAT TYPE AND DATES COVERED } \\
\text { Final Contractor Report }\end{array}$ \\
\hline
\end{tabular}

4. TITLE AND SUBTITLE

Measurement of Noise Produced by a Plasma Contactor Operating in Ground Based Facilities

6. AUTHOR(S)

Steve Snyder

7. PERFORMING ORGANIZATION NAME(S) AND ADDRESS(ES)

Colorado State University

Department of Mechanical Engineering

Fort Collins, Colorado 80523-1374

\section{FUNDING NUMBERS}

WU-478-43-10

G-NAG3-1700

9. SPONSORING/MONITORING AGENCY NAME(S) AND ADDRESS(ES)

10. SPONSORING/MONITORING AGENCY REPORT NUMBER

National Aeronautics and Space Administration

Lewis Research Center

Cleveland, Ohio 44135-3191

NASA CR-198438

11. SUPPLEMENTARY NOTES

Project Manager, Joel T. Galofaro, Power Technology Division, NASA Lewis Research Center, organization code 5470, (216) 433-2294.

12a. DISTRIBUTION/AVAILABILITY STATEMENT 12b. DISTRIBUTION CODE

Unclassified - Unlimited

Subject Category 14

This publication is available from the NASA Center for AeroSpace Information, (301) 621-0390.

13. ABSTRACT (Maximum 200 words)

Methods to measure electric-field fluctuations accurately in a plasma with an active monopole antenna are described. It is shown that the conductive surfaces of the antenna must be adequately isolated from the ambient plasma and that the monopole must be sufficiently short to avoid antenna amplifier saturation. Experimental results illustrate that the noise produced by plasma contactor operation and sensed by the antenna is due to plasma phenomena and is not induced by laboratory power supplies. A good correlation is shown between the current fluctuations in the contactor electrical circuit and the noise detected by the antenna. A large body of experimental data support the conclusion that the majority of noise sensed by the antenna at frequencies less than $1 \mathrm{MHz}$ is due to current fluctuations (electrostatic waves) in the plasma adjacent to the antenna and not to electromagnetic wave radiation. Caution is suggested when comparing antenna noise measurements to conventional specifications for radiated emissions.

14. SUBJECT TERMS 15. NUMBER OF PAGES

Plasma contactor; Electrical noise measurements; Conducted and radiated emissions 16. PRICE CODE

17. SECURITY CLASSIFICATION OF REPORT

Unclassified
18. SECURITY CLASSIFICATION OF THIS PAGE

Unclassified
19. SECURITY CLASSIFICATION OF ABSTRACT Unclassified 